\title{
Estimation of scan-gap limits on phase delay connections in Delta VLBI observations based on the phase structure function at a short time period
}

\author{
Tetsuro Kondo ${ }^{1,2}$, Thomas Hobiger ${ }^{1,3}$, Mamoru Sekido $^{1}$, Ryuichi Ichikawa ${ }^{1}$, \\ Yasuhiro Koyama ${ }^{1}$, and Hiroshi Takaba ${ }^{4}$ \\ ${ }^{1}$ Kashima Space Research Center, National Institute of Information and Communications Technology, \\ 893-1 Hirai, Kashima, Ibaraki 314-8501, Japan \\ ${ }^{2}$ Ajou University, San 5, Woncheon-dong, Yeongtong-gu, Suwon 442-749, Korea \\ ${ }^{3}$ Institute of Geodesy and Geophysics, Vienna University of Technology, \\ Gusshausstrasse 27-29 E128, A-1040 Wien, Austria \\ ${ }^{4}$ Gifu University, 1-1 Yanagido, Gifu-shi, Gifu 501-1193, Japan
}

(Received August 21, 2007; Revised August 28, 2008; Accepted October 15, 2008; Online published March 3, 2009)

\begin{abstract}
The maximum scan-gap length which connects phase delays from scan to scan over a gap is an important issue in Delta Very Long Baseline Interferometry (D-VLBI), and it is affected by delay fluctuations caused by the wet troposphere. It has recently become possible to obtain near real-time fringe phases by using an e-VLBI technique that realizes real-time VLBI by connecting stations through high-speed Internet. Such real-time VLBI raises the possibility of dynamic D-VLBI scheduling, which changes scan and gap length dynamically according to the weather condition of the date. We have investigated this possibility by using phase structure functions obtained from continuous VLBI observations at S- and X-bands for 1-2 h at the Kashima, Gifu, and Koganei stations (not real-time ones). Five VLBI sessions were conducted during this study between March and July 2006 under different weather conditions. At first a simple method was developed to evaluate phase connectivity from a phase structure function. A model was also proposed to estimate a phase-structure function at longer time periods from a short time period. Finally, an available gap length was estimated using the model. Our results show that it is possible to estimate an available scan gap length by using a structure function at a time period of $10 \mathrm{~s}$. This suggests that it is possible to control scan length and gap length dynamically in order to achieve the best performance of D-VLBI observations.
\end{abstract}

Key words: Delta VLBI, phase structure function, phase connection.

\section{Introduction}

Delta (or differential) Very Long Baseline Interferometry (D-VLBI) is a technique which accurately measures the angular separation between two nearby celestial radio sources by observing each source alternately. Common error sources, such as those introduced by receiving systems, clocks, propagating media, and station locations, are nearly cancelled by differences in observed delays between sources. If the position of one source is known well, the position of the other source can be measured through the wellknown source position. Since the late 1970's (e.g., Brunn et al., 1978; Christensen et al., 1980), this technique has been used for the navigation of deep-space spacecrafts (SC) by alternately observing SC and a nearby extragalactic radio source (EGRS). Since an antenna tracks an SC and an EGRS alternately, gaps in each data stream are inevitable. Consequently, the fringe phase of each data segment (scan) has an ambiguity with multiples of $2 \pi$. When observations are carried out at a narrow band width, such as in the case of

Copyright (c) The Society of Geomagnetism and Earth, Planetary and Space Sciences (SGEPSS); The Seismological Society of Japan; The Volcanological Society of Japan; The Geodetic Society of Japan; The Japanese Society for Planetary Sciences; TERRAPUB receiving carrier signals emitted from an SC, it is important to connect the fringe phases of corresponding scans without an ambiguity of $2 \pi$ multiples in order to improve the accuracy of the measurements. Wu (1979) developed a scheme to connect fringe phases among consecutive scans based on the iterative adjustment of integer ambiguity numbers. He applied the method to D-VLBI observations of Voyager 1 and OJ287 and concluded that when there was no sizable localized phase fluctuation (mainly due to the atmospheric disturbances), the scheme connected VLBI fringe phases faultlessly. Both scan and gap lengths were about $5 \mathrm{~min}$ in his study. A limitation in the gap lengths was not mentioned in his paper. However, as long as narrow-band carrier signals from an SC are received in D-VLBI, the phase ambiguity problem is inevitable. Hence, this problem has led to the development of a new technique aimed at abtaining an unambiguous delay from SC measurements by adopting bandwidth synthesis (Rogers, 1970) to a number of tones emitted from an SC spanned over several tens of megahertz, which depends on the design of the SC's telemetry system. Such unambiguous delay is called differential oneway range (DOR), and D-VLBI that has adopted DOR is termed $\triangle \mathrm{DOR}$ (e.g., Thornton and Border, 2003). Using an error budget analysis of the $\triangle \mathrm{DOR}$, Border and Koukos 
Table 1. Session summary.

\begin{tabular}{|c|c|c|c|c|c|}
\hline Date & Time & Source & Stations & Weather & Elevation at middle (deg) \\
\hline \multirow{2}{*}{ March 16, 2006} & \multirow{2}{*}{ 12:00-13:00 UT } & \multirow{2}{*}{$3 \mathrm{C} 273 \mathrm{~B}$} & Kashima $34 \mathrm{~m}$ & rainy & 36.2 \\
\hline & & & Gifu 11 m & rainy & 33.6 \\
\hline \multirow{2}{*}{ April 19, 2006} & \multirow{2}{*}{ 13:00-15:00 UT } & \multirow{2}{*}{$3 \mathrm{C} 273 \mathrm{~B}$} & Kashima $34 \mathrm{~m}$ & cloudy & 54.6 \\
\hline & & & Gifu 11 m & cloudy & 55.9 \\
\hline \multirow{2}{*}{ May 10, 2006} & \multirow{2}{*}{ 13:00-15:00 UT } & \multirow{2}{*}{$3 \mathrm{C} 273 \mathrm{~B}$} & Kashima $34 \mathrm{~m}$ & cloudy & 45.3 \\
\hline & & & Gifu $11 \mathrm{~m}$ & rainy & 47.9 \\
\hline \multirow{2}{*}{ May 11, 2006} & \multirow{2}{*}{ 13:00-15:00 UT } & \multirow{2}{*}{$3 \mathrm{C} 273 \mathrm{~B}$} & Kashima $34 \mathrm{~m}$ & cloudy & 44.7 \\
\hline & & & Gifu 11 m & fair & 47.3 \\
\hline \multirow{3}{*}{ July 21, 2006} & \multirow{3}{*}{ 15:00-17:00 UT } & \multirow{3}{*}{$3 \mathrm{C} 454.3$} & Kashima $34 \mathrm{~m}$ & cloudy & 61.2 \\
\hline & & & Gifu $11 \mathrm{~m}$ & rainy & 58.8 \\
\hline & & & Koganei $11 \mathrm{~m}$ & light rain & 60.5 \\
\hline
\end{tabular}

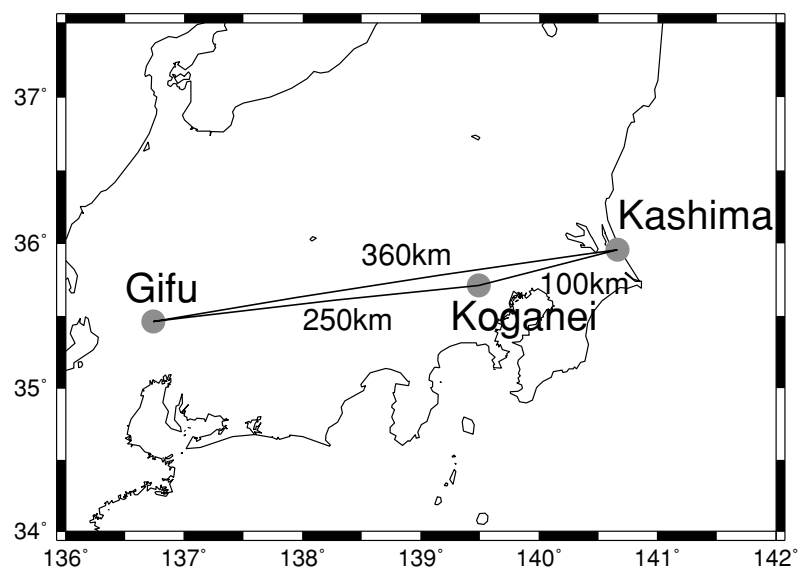

Fig. 1. Station locations.

Table 2. Observation characteristics.

\begin{tabular}{ll}
\hline Sampler & K5/VSSP (1 unit) \\
\hline Sampler mode & $4 \mathrm{MHz}, 1$ bit, $4 \mathrm{ch}$ \\
\hline RF frequency & $\mathrm{CH} 1: 8234.99 \mathrm{MHz}, \mathrm{CH} 2: 8534.99 \mathrm{MHz}$ \\
& $\mathrm{CH} 3: 2269.99 \mathrm{MHz}, \mathrm{CH} 4: 2344.99 \mathrm{MHz}$ \\
\hline Video bandwidths & $2 \mathrm{MHz}$ \\
\hline Volume of data/station & $7.2 \mathrm{~GB} /$ hour \\
\hline
\end{tabular}

(1994) demonstrated that the overall $\triangle D O R$ measurement accuracy is about $0.23 \mathrm{~ns}$, which corresponds to an angular position accuracy of about 9 nrad (nanoradian) for a projected baseline length of $8,000 \mathrm{~km}$.

Recently, the position measurements of NOZOMI, which is a Japanese Mars explorer (Yamamoto and Tsuruda, 1998), and HAYABUSA, which is a Japanese mission for near-Earth-asteroid sample return (Fujiwara et al., 1999), were carried out in Japan by means of D-VLBI (Ichikawa et al., 2004; Sekido et al., 2004; Ichikawa et al., 2006). The D-VLBI technique is also used for studying the lunar gravity field in the Japanese lunar explorer SELENE (KAGUYA) (Kono et al., 2003; Kikuchi et al., 2004). In the SELENE experiment, radio signals dedicated to D-VLBIlike $\triangle \mathrm{DOR}$ are emitted from an SC. However in the NOZOMI and HAYABUSA experiments, narrow-band signals emitted from an SC were observed in order to investigate the possibility of the orbit determination without the use of signals dedicated to the $\triangle \mathrm{DOR}$, and the connection of fringe phases among consecutive scans was again recognized as an important parameter to achieve a delay measurement accuracy equal to that of the $\triangle \mathrm{DOR}(0.23 \mathrm{~ns})$. Because the fluctuated fringe phases, mainly due to the wet troposphere, limit the gap length over which fringe phases can be connected, the antenna switching time should be less than this time span. Therefore, the maximum limit of the gap length as well as source strength are a critical parameters for promoting D-VLBI successfully. A gap length limit should depend on weather condition and, therefore, vary not only spatially but also temporally. It is therefore important to dynamically determine the limit of the gap length for achieving the best performance of D-VLBI observation under any weather conditions. Statistical characteristics of atmospheric phase fluctuations have been investigated using radio interferometry, including VLBI (e.g., Rogers and Moran, 1981; Rogers et al., 1984; Kasuga et al., 1986; Treuhaft and Lanyi, 1987; Liu et al., 2005). Beasley and Conway (1995) predicted a critical switching time based on their analysis of structure functions to $160-440 \mathrm{~s}$ at a frequency of $8.4 \mathrm{GHz}$ and $25-30 \mathrm{~s}$ at $43 \mathrm{GHz}$ at zenith angles less than $60^{\circ}$. A switching time of $300 \mathrm{~s}$ is therefore recommended for phase referencing observation of the VLA and VLBA for frequencies $1.4-8.4 \mathrm{GHz}$ under typical atmospheric conditions (Wrobel et al., 2000). The e-VLBI technique is developing rapidly (e.g., Koyama et al., 2006; Whitney and Ruszczyk, 2006), enabling scientists to obtain near real-time fringe phases. If we know the gap limit using such a real-time observation, we could control scan length and gap length (i.e., switching time) dynamically in order to achieve the best performance of D-VLBI observations. To this end, we have investigated the possibility of dynamic scheduling of D-VLBI observations using data obtained from a series of VLBI sessions with the aim of investigating fringe phase fluctuations.

We first propose a simple method to evaluate the scangap limit based on the error analysis of linear-fitted data using a phase structure function, and then we compare the results obtained by this simple method with those obtained from actual linear fitting of fringe phases. We next estimate a phase structure function on a longer time range from an observation of a short time period and then evaluate a scan-gap limit using the structure function estimated in this way. The results are compared with those obtained from 

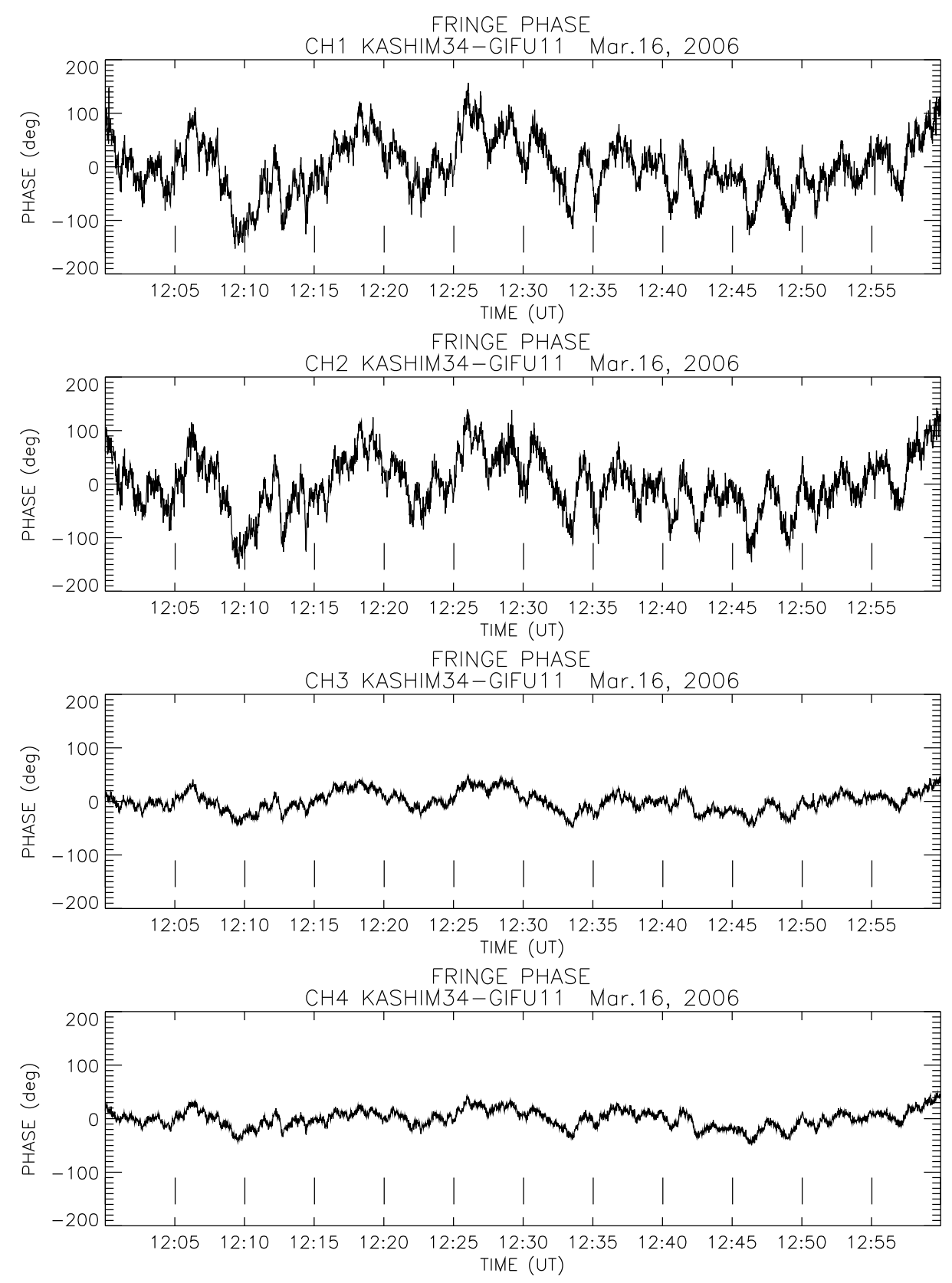

Fig. 2. Examples of fringe phase data after the connection of sub-observations and the removal of linear and second order trends for observations on March 16, 2006. Boundaries of sub-observations are displayed by short vertical bars, so that time between bars denotes 5 min. Frequencies from top to bottom are $8234.99 \mathrm{MHz}, 8534.99 \mathrm{MHz}, 2269.99 \mathrm{MHz}$, and 2344.99 MHz, respectively.

actual linear fitting of fringe phases, and the validity of the proposed method is discussed.

\section{Observations and Correlation Processing}

Five VLBI sessions were conducted to investigate the relations between scan length and gap length between March and July, 2006, as summarized in Table 1. The Kashima 34-m antenna and Gifu 11-m antenna were used in all sessions, but the Koganei 11-m antenna was used only in the last session. At all stations, a hydrogen maser oscillator was used to provide the system reference signals. The locations of stations are shown in Fig. 1.

A strong source (either $3 \mathrm{C} 273 \mathrm{~B}$ or $3 \mathrm{C} 454.3$ ) was observed at each session for 1 or $2 \mathrm{~h}$ continuously using four channels of geodetic-VLBI backend outputs. The RF fre- quencies were set to 8234.99 and $8584.99 \mathrm{MHz}$ in X-band and 2269.99 and $2344.99 \mathrm{MHz}$ in S-band, and the video bandwidth was set to $2 \mathrm{MHz}$. Four-channel video signals were converted into 1-bit digital signals at a sampling frequency of $4 \mathrm{MHz}$ using the K5/VSSP system (Kondo et al., 2003), which results in a data rate of 16 Mbps (Mega bits per second) per station. Sampled data were stored on a hard disk in real time. Details of the condition of data acquisition are summarized in Table 2.

One continuous observation was divided into a number of 5-min sub-observations to make data transfer and correlation processing easier. There is no gap between subobservations in an observation schedule itself, but a gap of a few seconds did occur between sub-observations due to the overhead of the data acquisition system. 


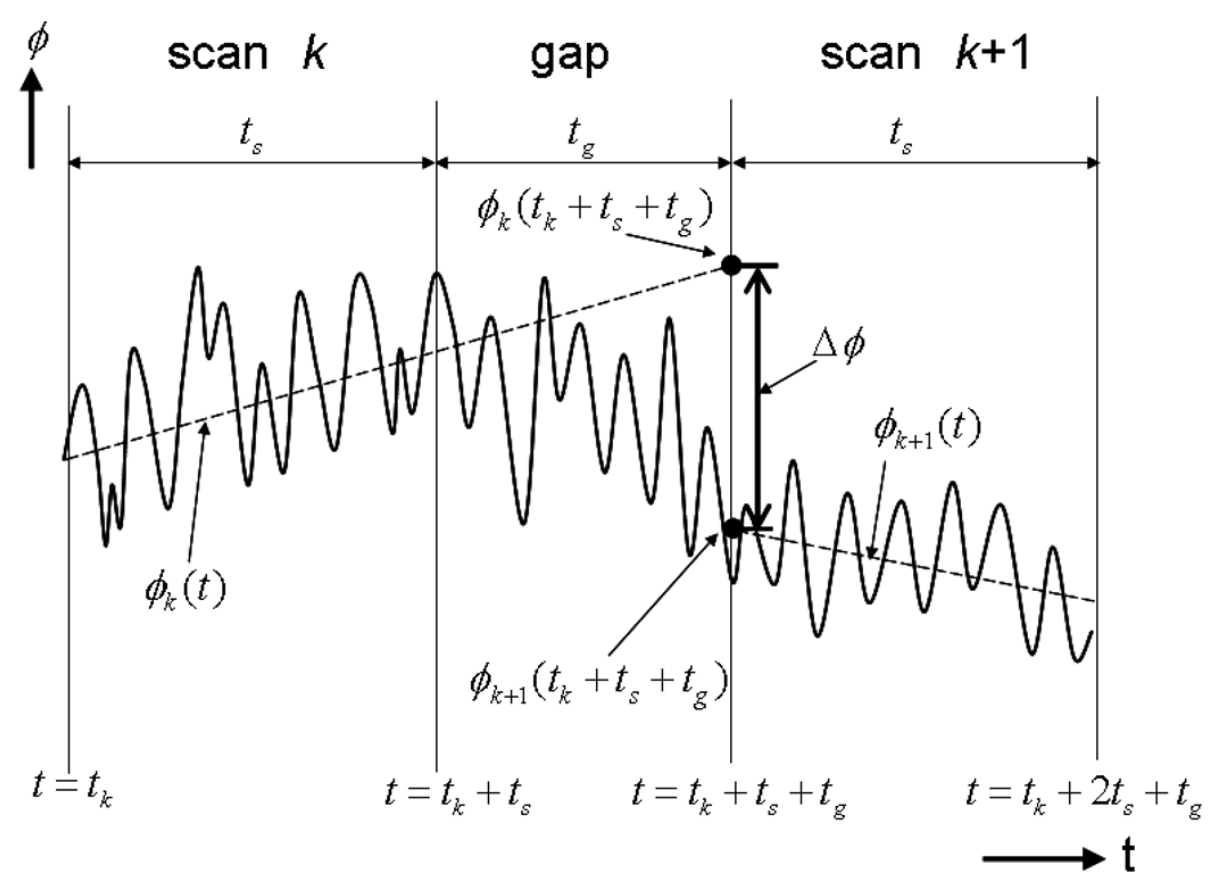

Fig. 3. Principle of the phase connection over a scan gap. Phases can be connected without ambiguities in case of $|\Delta \phi|<180^{\circ}$.

However it is easy to connect data over such a small gap, so that we can regard a series of sub-observations as one continuous observation for our analyses described later.

After all observations of a session were finished, the data of Gifu and Koganei were transferred to Kashima through the Internet. Correlation processing was carried out using a software correlator dedicated to the processing of geodetic VLBI data (Kondo et al., 2004). The software correlator can process data on each scan basis with a flexible combination of the parameters lag lengths and unit integration period. We processed the data in the same fashion as that of normal-geodetic VLBI, i.e., 32 lags and a unit integration period of $1 \mathrm{~s}$. A physical model used for the calculation of a-priori delay and delay rate was identical to that used for geodetic VLBI to ensure that the model has sufficient accuracy for our study. A-priori delay and delay rates were calculated for each sub-observation. Discrepancies at the boundaries of sub-observations are kept less than $0.01 \mathrm{~ns}$ for delay and $2 \times 10^{-14} \mathrm{~s} / \mathrm{s}$ for delay rate, corresponding to a fringe phase of $28.8^{\circ}$ and a fringe rate of $0.00016 \mathrm{~Hz}$ at $8 \mathrm{GHz}$. After correlation processing, residual delay and delay rate are determined every sub-observation by a coarse search, which calculates a so-called coarse search function. This is a function of trial delay and delay rate to find out the residual delay and delay rate which maximize the amplitude of the function (see, for example, Takahashi et al. (2000) for details of this processing). Observed delay and delay rate at an epoch of each sub-observation are computed by adding these residuals to the a-priori values. Therefore, as long as the discrepancy in the a-priori values is small enough to keep coherency in a unit integration period, it will not affect the observed delay and delay rate. Moreover, the model has sufficient accuracy to keep the position of correlation peak within a lag $(0.25 \mu \mathrm{s})$ during the entire session, i.e., 1-2 h. Figure 2 shows examples of fringe phase data after sub-observation connection and removal of linear and second order trend for the observation on May 11, 2006. Boundaries of sub-observations are displayed by short vertical bars in the Fig. 2.

\section{Data Analysis}

\subsection{Estimation of available scan-gap length from a phase structure function}

A simple method has been developed to evaluate an available scan-gap length using a phase structure function. Figure 3 shows the principle of phase connection between consecutive scans (scan numbers are $k$ and $k+1$, and scan length $=t_{\mathrm{s}}$ ) separated by a gap (length $=t_{\mathrm{g}}$ ). A phase at the starting time of scan $k+1$ (i.e., $t=t_{k}+t_{\mathrm{s}}+t_{\mathrm{g}}$ ) is estimated as an extrapolated phase using a linear fitting of data at scan $k$ spanning $t=t_{k}$ to $t_{k}+t_{\mathrm{s}}$.

The phase is then compared with a phase at $t=t_{k}+t_{\mathrm{s}}+t_{\mathrm{g}}$ estimated from a linear fitting in the scan $k+1$.

If the difference between these phases is smaller than $180^{\circ}$, phases are considered to be connected without ambiguities. Thus, the variance of these phase errors provides information on the connectivity of phases.

A line fitted to observed phase $\phi(t)$ for scan $k$ is given by

$$
\phi_{k}(t)=a_{k} t+b_{k}
$$

where $k$ is an index to represent scan number, and $a_{k}$ and $b_{k}$ are fitting coefficients for scan $k$. Since $\phi_{k}(t)$ is a linear fitted line to $\phi(t)$,

$$
\phi(t)=\phi_{k}(t)+\varepsilon_{k}(t)
$$

where $\varepsilon_{k}(t)$ is a fitting residual at $t$. An extrapolated phase at $t=t_{k}+t_{\mathrm{s}}+t_{\mathrm{g}}$ can be expressed using Eq. (1) as

$$
\begin{aligned}
\phi_{k}\left(t_{k}+t_{\mathrm{s}}+t_{\mathrm{g}}\right) & =a_{k}\left(t_{k}+t_{\mathrm{s}}+t_{\mathrm{g}}\right)+b_{k} \\
& =\phi_{k}\left(t_{k}+t_{\mathrm{s}}\right)+a_{k} t_{\mathrm{g}} .
\end{aligned}
$$




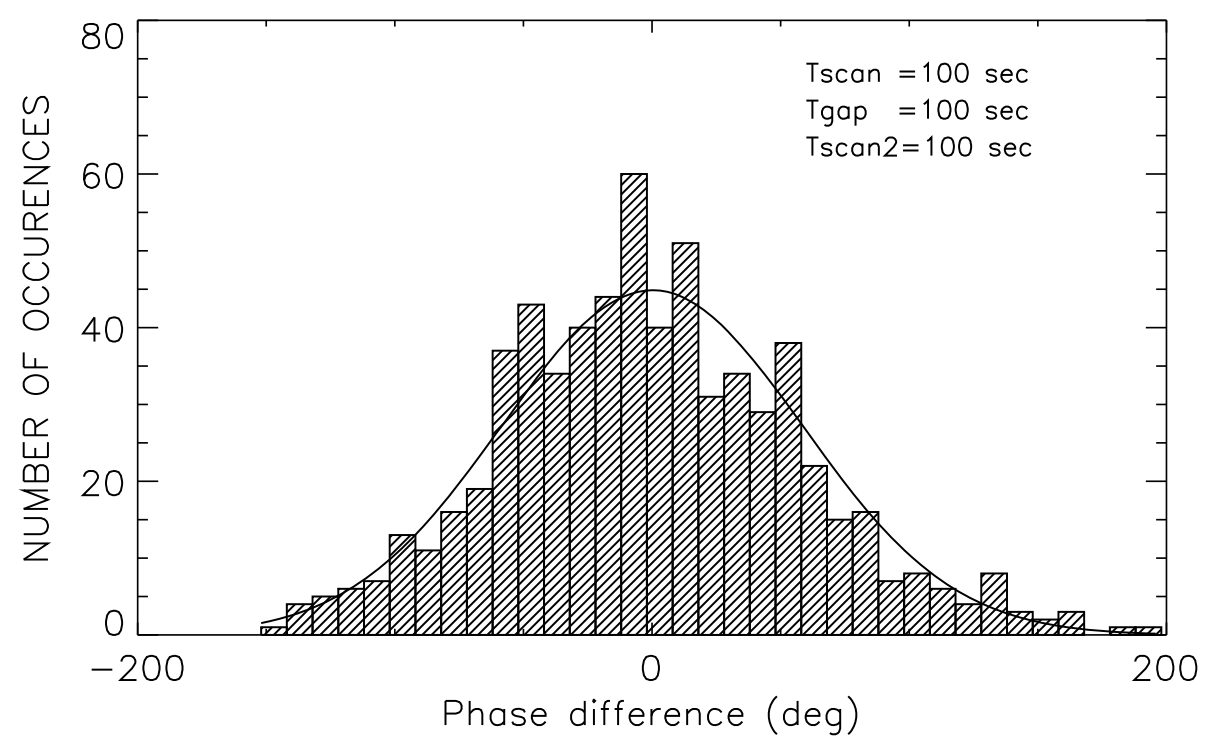

Fig. 4. An example histogram of $\Delta \phi$ for the case of scan length $=100 \mathrm{~s}$ and gap length $=100 \mathrm{~s}$ for $8234.99 \mathrm{MHz}$ data for the session on May 11 , 2006. Standard deviation is $58.5^{\circ}$.

Using Eq. (2), Eq. (3) can be rewritten as

$$
\phi_{k}\left(t_{k}+t_{\mathrm{s}}+t_{\mathrm{g}}\right)=\phi\left(t_{k}+t_{\mathrm{s}}\right)-\varepsilon_{k}\left(t_{k}+t_{\mathrm{s}}\right)+a_{k} t_{\mathrm{g}}
$$

Using Eq. (2), we also obtain

$$
\phi_{k+1}\left(t_{k}+t_{\mathrm{s}}+t_{\mathrm{g}}\right)=\phi\left(t_{k}+t_{\mathrm{s}}+t_{\mathrm{g}}\right)-\varepsilon_{k+1}\left(t_{k}+t_{\mathrm{s}}+t_{\mathrm{g}}\right) \text {. }
$$

Hence using Eqs. (4) and (5), the difference between $\phi_{k}\left(t_{k}+t_{\mathrm{s}}+t_{\mathrm{g}}\right)$ and $\phi_{k+1}\left(t_{k}+t_{\mathrm{s}}+t_{\mathrm{g}}\right)$ is given as

$$
\begin{aligned}
\Delta \phi= & \phi_{k}\left(t_{k}+t_{\mathrm{s}}+t_{\mathrm{g}}\right)-\phi_{k+1}\left(t_{k}+t_{\mathrm{s}}+t_{\mathrm{g}}\right) \\
= & {\left[\phi\left(t_{k}+t_{\mathrm{s}}\right)-\phi\left(t_{k}+t_{\mathrm{s}}+t_{\mathrm{g}}\right)\right]+a_{k} t_{\mathrm{g}} } \\
& -\left[\varepsilon_{k}\left(t_{k}+t_{\mathrm{s}}\right)-\varepsilon_{k+1}\left(t_{k}+t_{\mathrm{s}}+t_{\mathrm{g}}\right)\right] .
\end{aligned}
$$

(6) where $\sigma_{a}$ and $\sigma_{b}$ are estimation errors for parameter $a$ and Therefore the variance can be expressed as

$$
\begin{aligned}
\left\langle(\Delta \phi)^{2}\right\rangle= & \left\langle\left[\phi\left(t_{k}+t_{\mathrm{s}}\right)-\phi\left(t_{k}+t_{\mathrm{s}}+t_{\mathrm{g}}\right)\right]^{2}\right\rangle+t_{\mathrm{g}}^{2}\left\langle a_{k}^{2}\right\rangle \\
& +\left\langle\left[\varepsilon_{k}\left(t_{k}+t_{\mathrm{s}}\right)-\varepsilon_{k+1}\left(t_{k}+t_{\mathrm{s}}+t_{\mathrm{g}}\right)\right]^{2}\right\rangle \\
& +2 t_{\mathrm{g}}\left\langle a_{k}\left[\phi\left(t_{k}+t_{\mathrm{s}}\right)-\phi\left(t_{k}+t_{\mathrm{s}}+t_{\mathrm{g}}\right)\right]\right\rangle \\
& -2 t_{\mathrm{g}}\left\langle a_{k}\left[\varepsilon_{k}\left(t_{k}+t_{\mathrm{s}}\right)-\varepsilon_{k+1}\left(t_{k}+t_{\mathrm{s}}+t_{\mathrm{g}}\right)\right]\right\rangle \\
& -2\left\langle\left[\phi\left(t_{k}+t_{\mathrm{s}}\right)-\phi\left(t_{k}+t_{\mathrm{s}}+t_{\mathrm{g}}\right)\right]\right. \\
& \left.\cdot\left[\varepsilon_{k}\left(t_{k}+t_{\mathrm{s}}\right)-\varepsilon_{k+1}\left(t_{k}+t_{\mathrm{s}}+t_{\mathrm{g}}\right)\right]\right\rangle
\end{aligned}
$$

where \langle\rangle denotes the operation of averaging. Since $a_{k}, \varepsilon_{k}$, and $\varepsilon_{k+1}$ are thought to be independent stochastic variables, the average of their cross-term will be small compared with other terms. Hence, we omit these cross terms and obtain

$$
\begin{aligned}
\left\langle(\Delta \phi)^{2}\right\rangle= & \left\langle\left[\phi\left(t_{k}+t_{\mathrm{s}}\right)-\phi\left(t_{k}+t_{\mathrm{s}}+t_{\mathrm{g}}\right)\right]^{2}\right\rangle+t_{\mathrm{g}}^{2}\left\langle a_{k}^{2}\right\rangle \\
& +\left\langle\varepsilon_{k}^{2}\left(t_{k}+t_{\mathrm{s}}\right)\right\rangle+\left\langle\varepsilon_{k+1}^{2}\left(t_{k}+t_{\mathrm{s}}+t_{\mathrm{g}}\right)\right\rangle .
\end{aligned}
$$

The effect of cross terms will be discussed later.

Now, as the scan length is the same for scan $k$ and $k+1$, $\left\langle\varepsilon_{k}^{2}\right\rangle$ and $\left\langle\varepsilon_{k+1}^{2}\right\rangle$ coincide with each other $\left(=\varepsilon^{2}\right)$. Moreover, using a phase structure function defined as

$$
\sigma^{2}(\tau)=\left\langle[\phi(t)-\phi(t+\tau)]^{2}\right\rangle,
$$

we obtain

$$
\left\langle(\Delta \phi)^{2}\right\rangle=\sigma^{2}\left(t_{\mathrm{g}}\right)+t_{\mathrm{g}}^{2}\left\langle a_{k}^{2}\right\rangle+2 \varepsilon^{2}
$$

Statistical characteristics of fringe phase fluctuations usually differ from a normal distribution on the time scale used in this study. However, we apply a fitting error based on a normal distribution variable to simplify the problem. The effect of this assumption will be discussed later. Hence $\varepsilon$ is given as

$$
\varepsilon^{2}=\frac{1}{4} t_{\mathrm{s}}^{2} \sigma_{a}^{2}+\sigma_{b}^{2}
$$

$b$. Hence, assuming $\left\langle a_{k}^{2}\right\rangle=\sigma_{a}^{2}$, we get

$$
\left\langle(\Delta \phi)^{2}\right\rangle=\sigma^{2}\left(t_{\mathrm{g}}\right)+\left(\frac{1}{2} t_{\mathrm{s}}^{2}+t_{\mathrm{g}}^{2}\right) \sigma_{a}^{2}+2 \sigma_{b}^{2}
$$

The second term of the right-hand side of Eq. (12) represents a propagated phase error over a gap due to the error of the phase changing rate.

Regarding a linear fitting, we consider the simplest case, that is a line fitting using phases only at $t=t_{k}$ and $t_{k}+t_{\mathrm{s}}$ and assuming that the variance of their phase difference has an error $\sigma_{a}^{2}$ that can be given by

$$
\sigma_{a}^{2}=\frac{\sigma^{2}\left(t_{\mathrm{s}}\right)}{t_{\mathrm{s}}^{2}}
$$

where $\sigma^{2}\left(t_{\mathrm{s}}\right)$ is a phase structure function at $t_{\mathrm{s}}$. The relation between a phase error due to thermal noise and signal-tonoise ratio (SNR) can be written as

$$
\sigma_{\phi}=\frac{1}{S N R_{\mathrm{u}}}
$$

where $S N R_{\mathrm{u}}$ is an SNR at a unit integration period at the correlation processing. Therefore, using Eq. (14), $\sigma_{b}^{2}$ is 

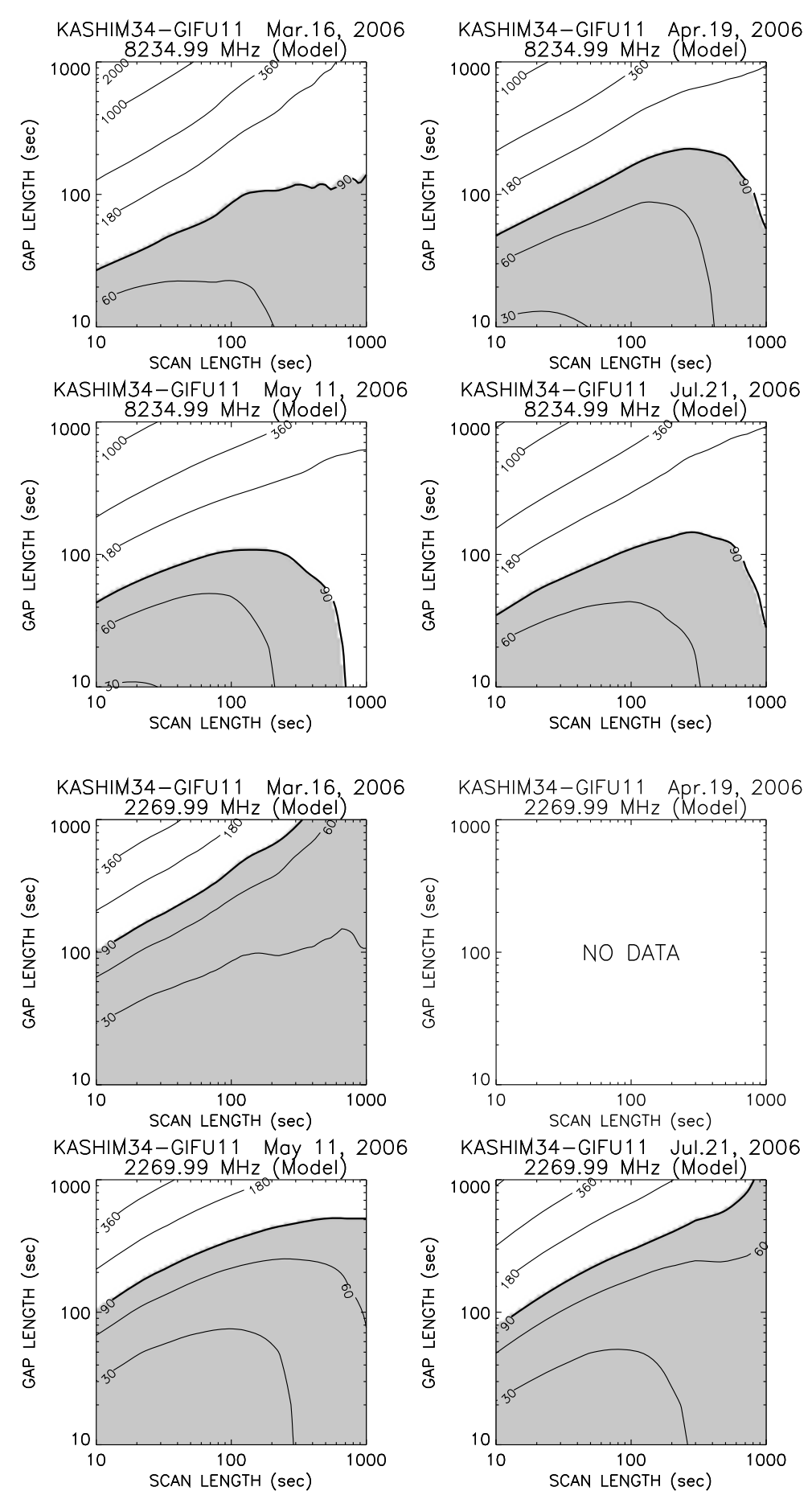
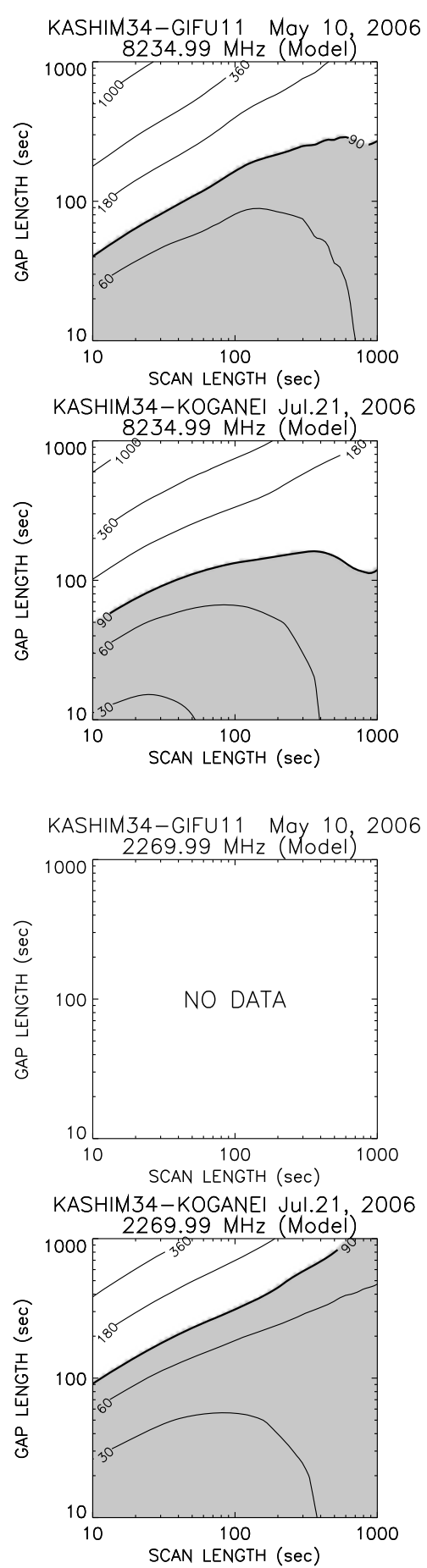

Fig. 5. Contour plots of standard deviations of $\Delta \phi$ on a scan and gap length plane obtained from phase structure functions for six baseline data from the five sessions carried out on March 16, April 19, May 20, May 21, and July 21, 2006, respectively. Upper six pannels are for 8234.99 MHz and lower six for $2269.99 \mathrm{MHz}$.

given as

$$
\sigma_{b}^{2}=\frac{\sigma_{\phi}^{2}}{t_{\mathrm{s}}}=\frac{1}{\left(S N R_{\mathrm{u}}\right)^{2} t_{\mathrm{s}}}=\frac{1}{(S N R)^{2}}
$$

where SNR denotes an SNR integrated over the whole scan length. Here, we assume that the only source of noise is thermal noise to simplify the problem. The effect of this assumption will be discussed later. Hence Eq. (12) can be rewritten using $S N R$ as

$$
\left\langle(\Delta \phi)^{2}\right\rangle=\sigma^{2}\left(t_{\mathrm{g}}\right)+\left(\frac{1}{2}+\frac{t_{\mathrm{g}}^{2}}{t_{\mathrm{s}}^{2}}\right) \sigma^{2}\left(t_{\mathrm{s}}\right)+\frac{2}{(S N R)^{2}} .
$$

Thus, if a phase structure function and SNR are given, we can evaluate the phase error variance from Eq. (16). On the other hand, $\sigma_{\phi}$ can also be expressed by using a phase structure function as

$$
\sigma_{\phi}^{2}=\frac{1}{2} \sigma^{2}\left(t_{\mathrm{u}}\right)
$$



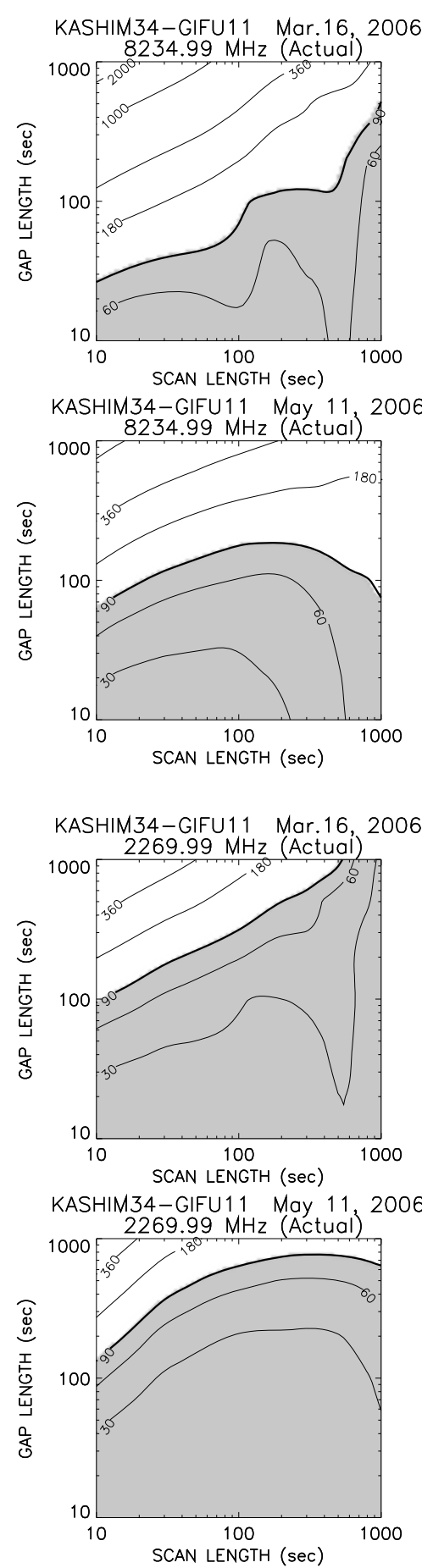
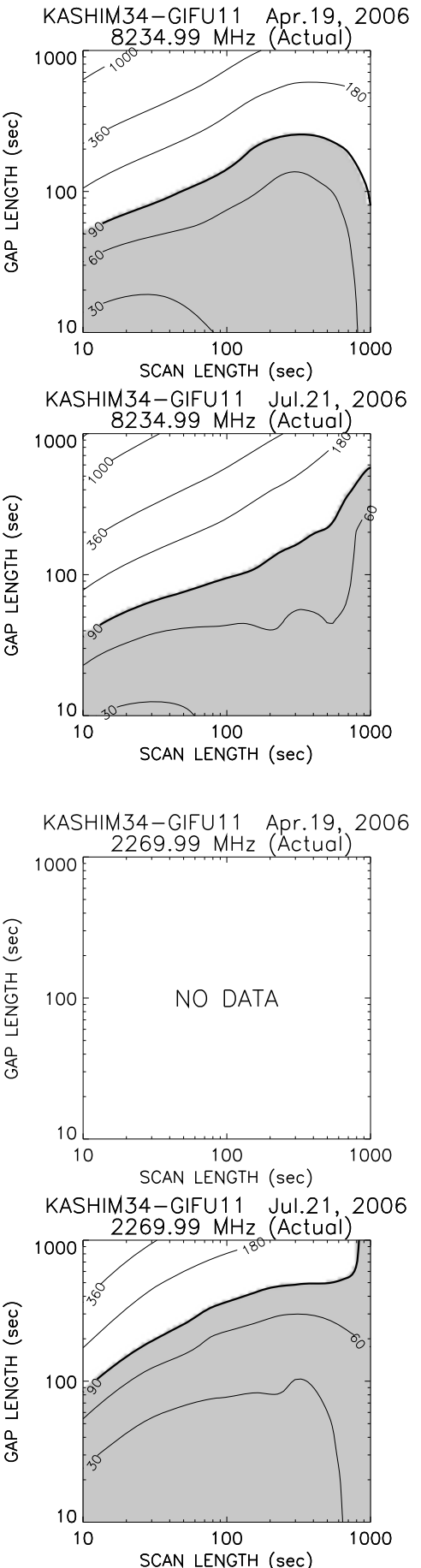
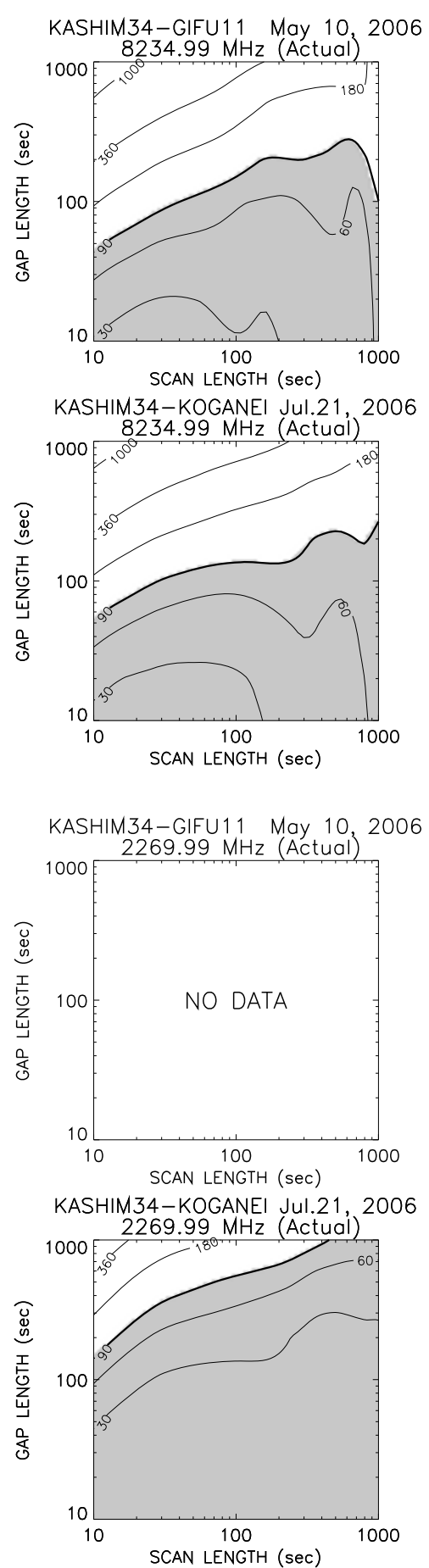

Fig. 6. Same as Fig. 5 but obtained from the actual linear fitting of phase data.

where $t_{\mathrm{u}}$ is a unit integration period ( $1 \mathrm{~s}$ in this study). simplified as follows

Applying this to Eq. (17), we get

$$
\left\langle(\Delta \phi)^{2}\right\rangle=\sigma^{2}\left(t_{\mathrm{g}}\right)+\left(\frac{1}{2}+\frac{t_{\mathrm{g}}^{2}}{t_{\mathrm{s}}^{2}}\right) \sigma^{2}\left(t_{\mathrm{s}}\right)+\frac{1}{t_{\mathrm{s}}} \sigma^{2}\left(t_{\mathrm{u}}\right)
$$

Equation (18) shows that we can evaluate the connectivity of fringe phases only from the phase structure function. In case of gap length = scan length, Eqs. (16) and (18) can be

$$
\begin{aligned}
\left\langle(\Delta \phi)^{2}\right\rangle & =\frac{5}{2} \sigma^{2}\left(t_{\mathrm{s}}\right)+\frac{2}{(S N R)^{2}} \\
& =\frac{5}{2} \sigma^{2}\left(t_{\mathrm{s}}\right)+\frac{1}{t_{\mathrm{s}}} \sigma^{2}\left(t_{\mathrm{u}}\right) .
\end{aligned}
$$

If an SNR integrated over the span length is high enough, we can neglect the SNR term. The variance then becomes

$$
\left\langle(\Delta \phi)^{2}\right\rangle=\frac{5}{2} \sigma^{2}\left(t_{\mathrm{s}}\right) .
$$



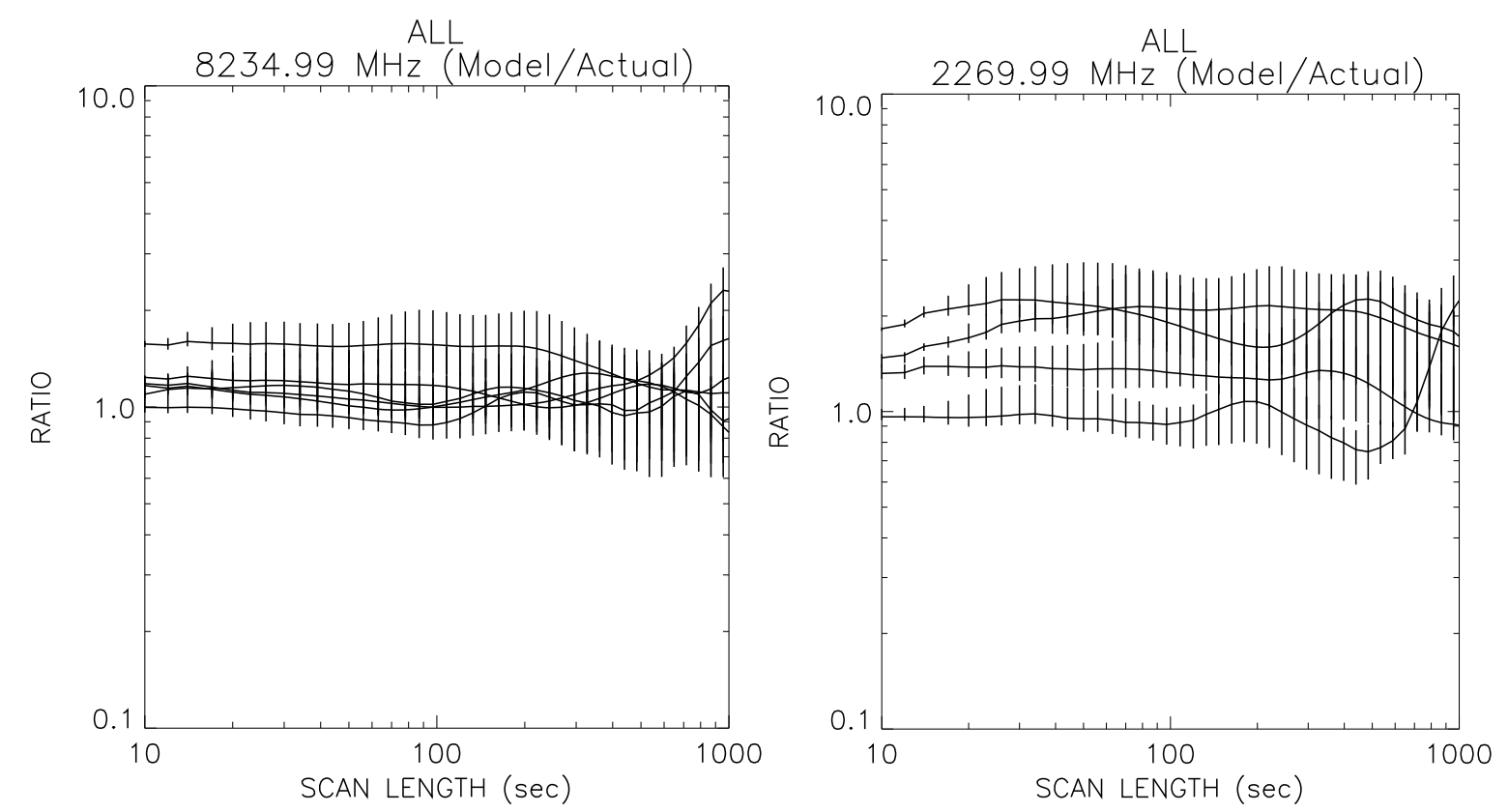

Fig. 7. Ratios of standard deviation of phase errors obtained from phase structure functions and those obtained from actual linear fitting for $8234.99 \mathrm{MHz}$ (left panel) and for $2269.99 \mathrm{MHz}$ (right panel). Averaged values over gap length between 10 and $1000 \mathrm{~s}$ are plotted for all sessions. Vertical bars denote the range between maximum and minimum of ratios.

In order to evaluate the simple method proposed above, the variance of $\Delta \phi$ is calculated for various combinations of scan length and gap length using an actual linear fitting of fringe phase data. Figure 4 shows an example histogram of phase differences and a Gaussian fitting curve obtained for the case of $t_{\mathrm{s}}=100 \mathrm{~s}$ and $t_{\mathrm{g}}=100 \mathrm{~s}$ using fringe phase data observed on May 11, 2006 at $8234.99 \mathrm{MHz}$. Standard deviations of $\Delta \phi$ (root of the variance) obtained by this way are compared with those obtained from phase structure functions, i.e., Eq. (18).

Observations at the S-band failed in two session carried out on April 19 and May 10, 2006, among the overall five sessions shown in Table 1 . We therefore excluded these Sband data in the analysis. The analysis period of the May 10 session was shortened to $1 \mathrm{~h}$ because of failure scans in the last 1-h period. Data from the Koganei-Gifu baseline of the July 21 session were also excluded due to insufficient SNR data, making it impossible to obtain reliable results.

Figure 5 shows contour plots of the standard deviations of $\Delta \phi$ in a coordinate system where the abscissa represents scan length and ordinate shows gap length the obtained from phase structure functions for data of six baselines from the five sessions. The upper six panels are for 8234.99 MHz and the lower six for 2269.99 MHz. Figure 6 shows the same contour plots as Fig. 5 but for standard deviations obtained from the actual linear fitting of the phase data. Shaded areas in the figures represent zones where the standard deviation is less than $90^{\circ}$, which means phases can be connected without ambiguities with a probability of larger than $95 \%$. For example, if we assume $90^{\circ}$ as a limit for connecting phases and we take $100 \mathrm{~s}$ for scan length, the gap length can be about $100 \mathrm{~s}$ for $8234.99 \mathrm{MHz}$ and about $200 \mathrm{~s}$ for $2269.99 \mathrm{MHz}$ for the March 16 data.

We can see that shaded areas in Fig. 5 coincide well with those in Fig. 6. Figure 7 shows their ratio averaged over gap lengths between 10 to $1000 \mathrm{~s}$ as a function of scan length, with the vertical bars indicating the range of ratio. Although ratios are averaged values, a ratio of unity can be interpreted as an indication that they coincide well with each other. Thus, we consider that the simple model used in this study to calculate standard deviations of $\Delta \phi$ well explains the condition of phase connection over a gap. This means that if a phase structure function is given, we can estimate an available scan-gap length. If we know a phase structure function on longer time periods from a shorter time range observation, we could control scan length and gap length dynamically in order to get the best performance of the D-VLBI observation. Therefore, we investigate here the possibility of estimating a phase structure function in longer time periods from a shorter time range observation.

\subsection{Estimation of a structure function at longer time periods}

According to the Kolmogorov turbulence theory (Tatarskii, 1961), variances of phase fluctuations due to the fluctuations of water vapor delay are proportional to $t^{5 / 3}$ at a short time scale and proportional to $t^{2 / 3}$ at a large time scale; these are called 3-D and 2-D turbulences, respectively. Transient time between $3-\mathrm{D}$ and 2-D turbulence is given by the thickness of the wet troposphere divided by mean wind velocity. According to Treuhaft and Lanyi (1987), the change from the short period to long period temporal behavior takes place near $125 \mathrm{~s}$, which corresponds to a thickness of $1 \mathrm{~km}$ divided by a mean wind velocity of $8 \mathrm{~m} / \mathrm{s}$.

We first investigate whether we can see these characteristics in our fringe phase data or not. In order to obtain a phase structure function contributed by atmospheric origin, we calculate a phase structure function defined as

$$
\sigma_{n}^{2}(\tau)=\sigma^{2}(\tau)-\sigma^{2}(0)
$$



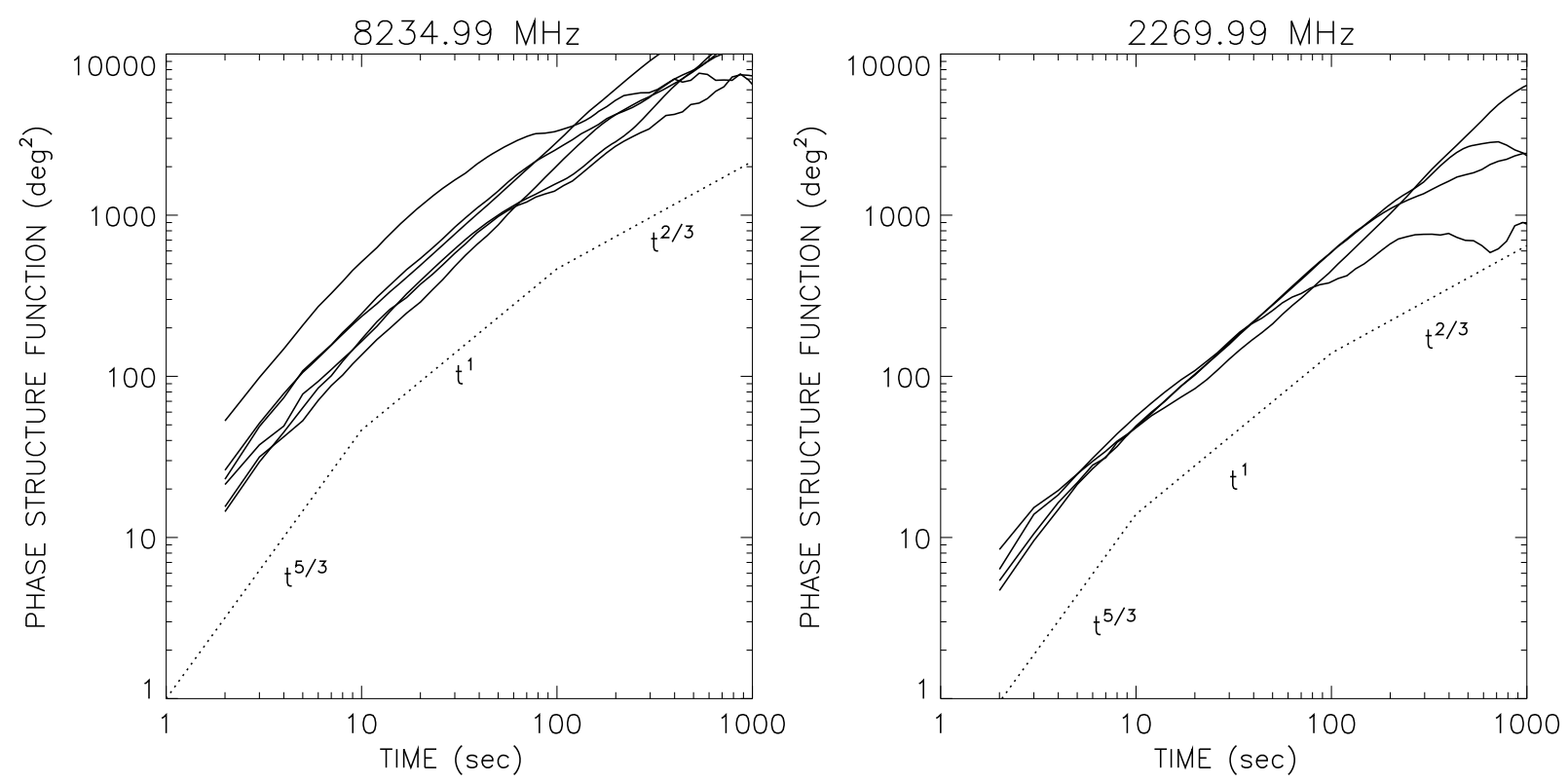

Fig. 8. Phase-structure-functions after removal of thermal phase noises for $8243.99 \mathrm{MHz}$ (left panel) and $2269.99 \mathrm{MHz}$ (right panel) for all sessions. Dotted lines show model slopes simulating Kolmogorov turbulences (see text for details).

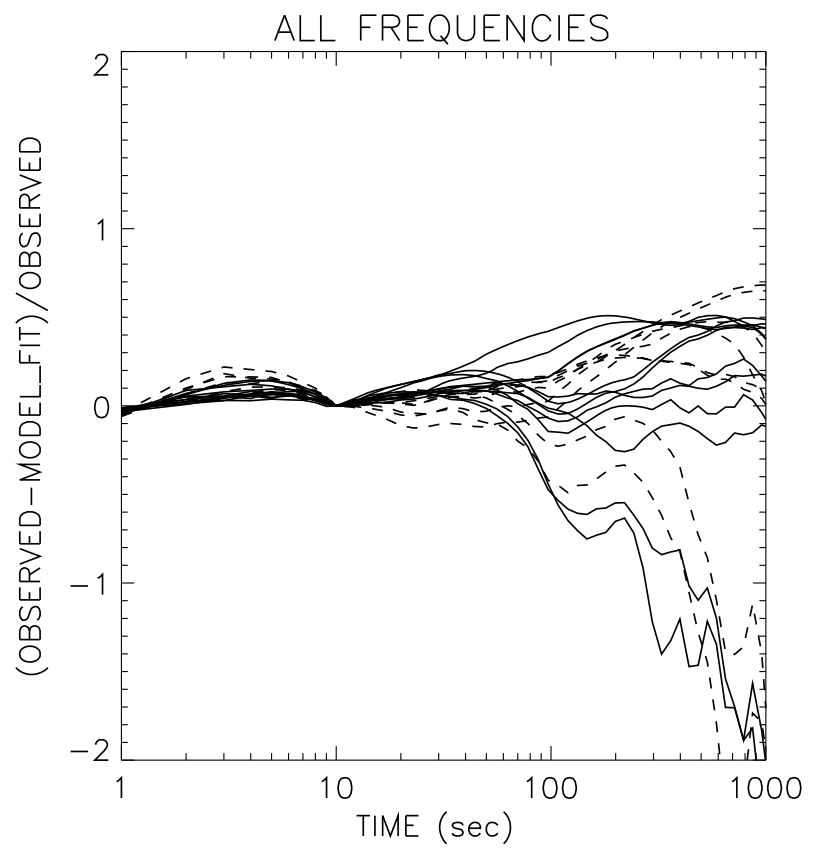

Fig. 9. Relative error of phase-structure-functions estimated from those at $\tau=10 \mathrm{~s}$ for all frequencies and for all sessions. Solid lines are for $\mathrm{X}$-band data and dashed lines for S-band.

where $\sigma^{2}(0)$ is the phase variance at the shortest time interval (i.e., $1 \mathrm{~s}$ in this study), assuming that this represents a phase noise caused by thermal noise (i.e., related to SNR). Hence, $\sigma_{n}^{2}(\tau)$ is thought to be of atmospheric origin, including some instrumentation noises as well as a thermal noise. Figure 8 shows $\sigma_{n}^{2}(\tau)$ at $8243.99 \mathrm{MHz}$ and $2269.99 \mathrm{MHz}$ for all sessions. As shown in this figure, $\sigma_{n}^{2}(\tau)$ seems to follow the characteristics of Kolmogorov turbulence, i.e., $\sigma_{n}^{2}(\tau)$ is proportional to $t^{5 / 3}$ for periods less than $10 \mathrm{~s}$ and is proportional to $t^{2 / 3}$ for periods longer than $100 \mathrm{~s}$. Although the absolute value of $\sigma_{n}^{2}(\tau)$ reflects weather conditions at every session, the slopes seem to be stable, in particular for periods less than $100 \mathrm{~s}$. Therefore, we model $\sigma_{n}^{2}(\tau)$ using a piecewise linear function on the log-log plot as follows,

$$
\hat{\sigma}_{n}^{2}(\tau)=C_{i} \tau^{B_{i}}
$$

with the following boundary condition,

$$
C_{i} t_{i+1}^{B_{i}}=C_{i+1} t_{i+1}^{B_{i+1}}
$$

where $i$ is an index representing the time range of $t_{i} \sim$ $t_{i+1}$ and $C_{i}$ is a parameter to be fitted for time range $t_{i}$. $B_{i}$ is a fixed parameter, i.e., $B_{i}$ is not estimated, but only $C_{i}$ is estimated. Therefore, if $\sigma_{n}^{2}(\tau)$ is given at a certain $\tau$, we can determine all $C_{i} \mathrm{~s}$ using the relation given by Eq. (24). This means that $\sigma_{n}^{2}(\tau)$ at a longer time scale can be inferred from that at a shorter time scale. Thus, if the fringe phase is observed in real-time, we can estimate an available scan-gap limit in real-time, and the most suitable scan length and scan gap for D-VLBI observations, which will depend on weather conditions during the observation, can be determined dynamically. Once a phase structure function contributed by atmosphere is inferred, an overall phase structure function can be given by adding an SNR phase noise as

$$
\hat{\sigma}^{2}(\tau)=2 \sigma_{S N R_{\mathrm{u}}}^{2}+\hat{\sigma}_{n}^{2}(\tau)
$$

where $\sigma_{S N R_{\mathrm{u}}}$ is a phase error due to a thermal noise of a unit integration period.

An actual procedure to obtain $\hat{\sigma}_{n}^{2}(\tau)$ is as follows. We use three time ranges, 1-10, 10-100, 100-1000 s, so that $t_{1}$, $t_{2}, t_{3}$, and $t_{4}$ are $1,10,100$, and 1000 , respectively. Parameter $C_{2}$, i.e., $C$ at the second time range, is first calculated using real $\sigma_{n}^{2}(\tau)$ observed for $\tau=10 \mathrm{~s}$. Parameters $C_{1}$ and $C_{3}$ are then calculated using Eq. (24). For parameter $B_{i}$ s for three time ranges, we fix these as $1.67(=5 / 3), 1.00$, and $0.67(=2 / 3)$, which is intended to simulate Kolmogorov 

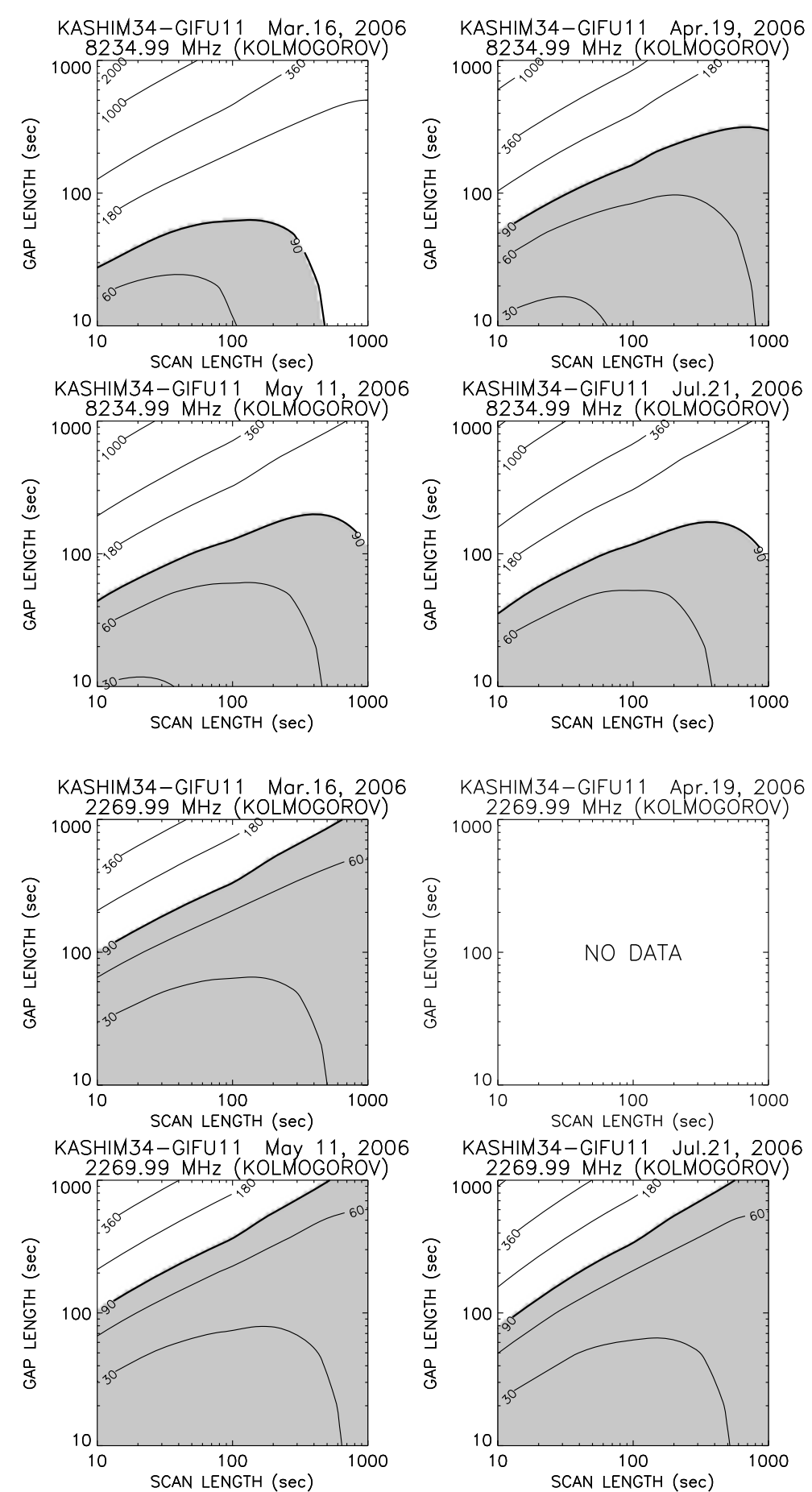

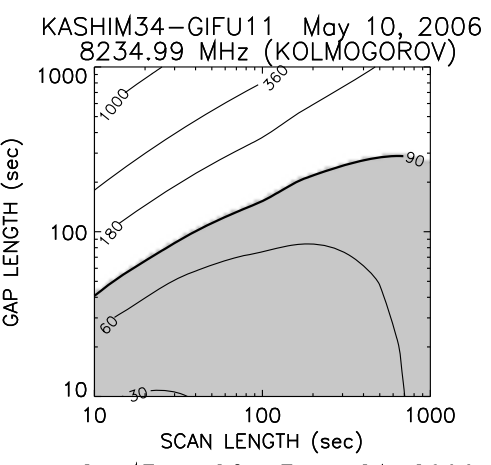

KASHIM34-KOGANEI Jul.21, 2006
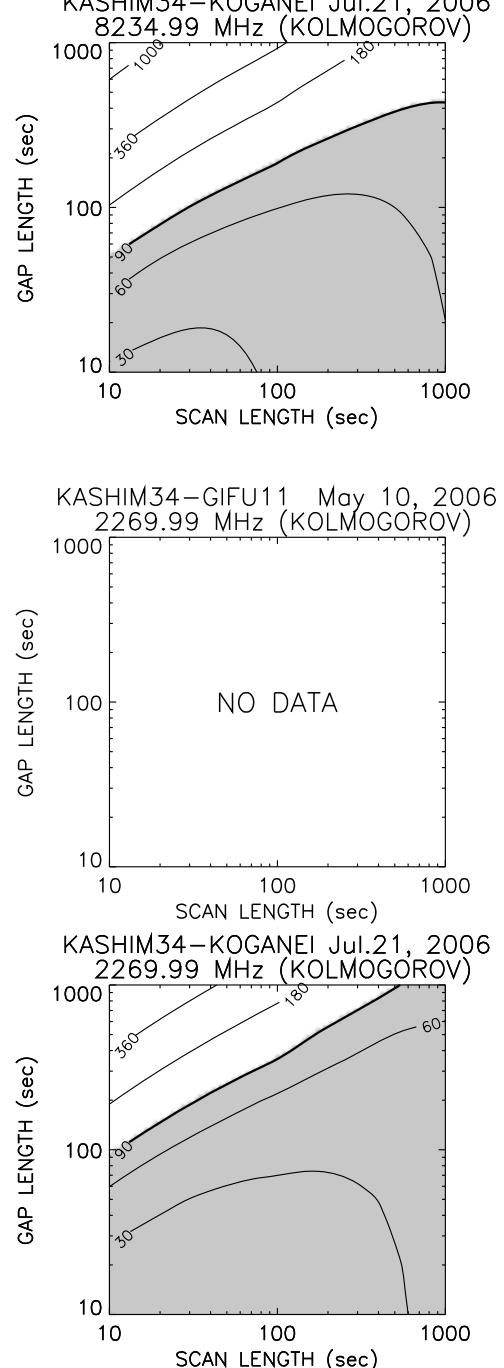

Fig. 10. Same as Fig. 5 but obtained by Kolmogorov model fitting. See text for details.

turbulences. Figure 9 shows relative errors of $\hat{\sigma}^{2}(\tau)$ estimated this way for all frequencies and for all sessions. We can then calculate standard deviations of $\Delta \phi$ using obtained $\hat{\sigma}^{2}(\tau)$. Results are shown in Fig. 10 .

\section{Results and Discussion}

Fringe phase data used in this study are those obtained after the removal of linear and second order trend for a whole observation period of 3600 or $7200 \mathrm{~s}$, in other words, fluctuations with a time scale longer than a few thousand seconds are smeared out. However, the time scale concerned in this study is shorter than $1000 \mathrm{~s}$, so that removal of the trend does not affect the results of this study.

Equation (18) calculates the variance of $\Delta \phi$ as a function of scan length and gap length from a phase structure function. It is derived from a simple model. Assumptions used in the model are summarized as follows.

A1) Neglecting cross-terms of $a_{k}, \varepsilon_{k}$, and $\varepsilon_{k+1}$ to derive Eq. (8) from Eq. (7);

A2) Use of a theoretical fitting error based on a normal distribution variable in Eq. (11) to simplify the problem;

A3) Use of phases only at $t=t_{k}$ and $t_{k}+t_{\mathrm{s}}$ to derive $\sigma_{a}^{2}$ in Eq. (13); 

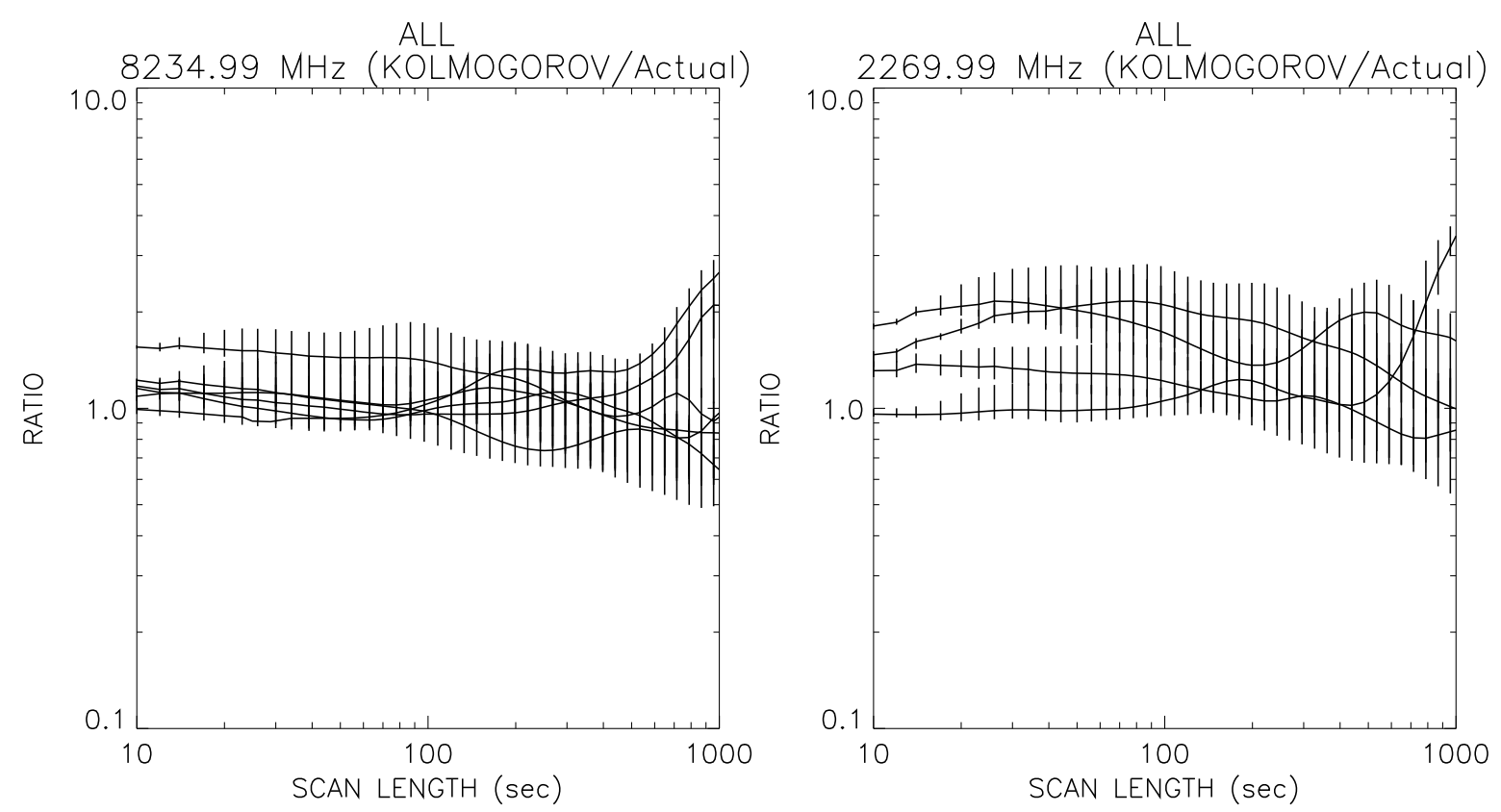

Fig. 11. Same as Fig. 7 but for ratios of standard deviation of phase errors obtained from phase structure functions obtained by the Kolmogorov model fitting and those obtained from actual linear fitting for $8234.99 \mathrm{MHz}$ (left panel) and for $2269.99 \mathrm{MHz}$ (right panel).
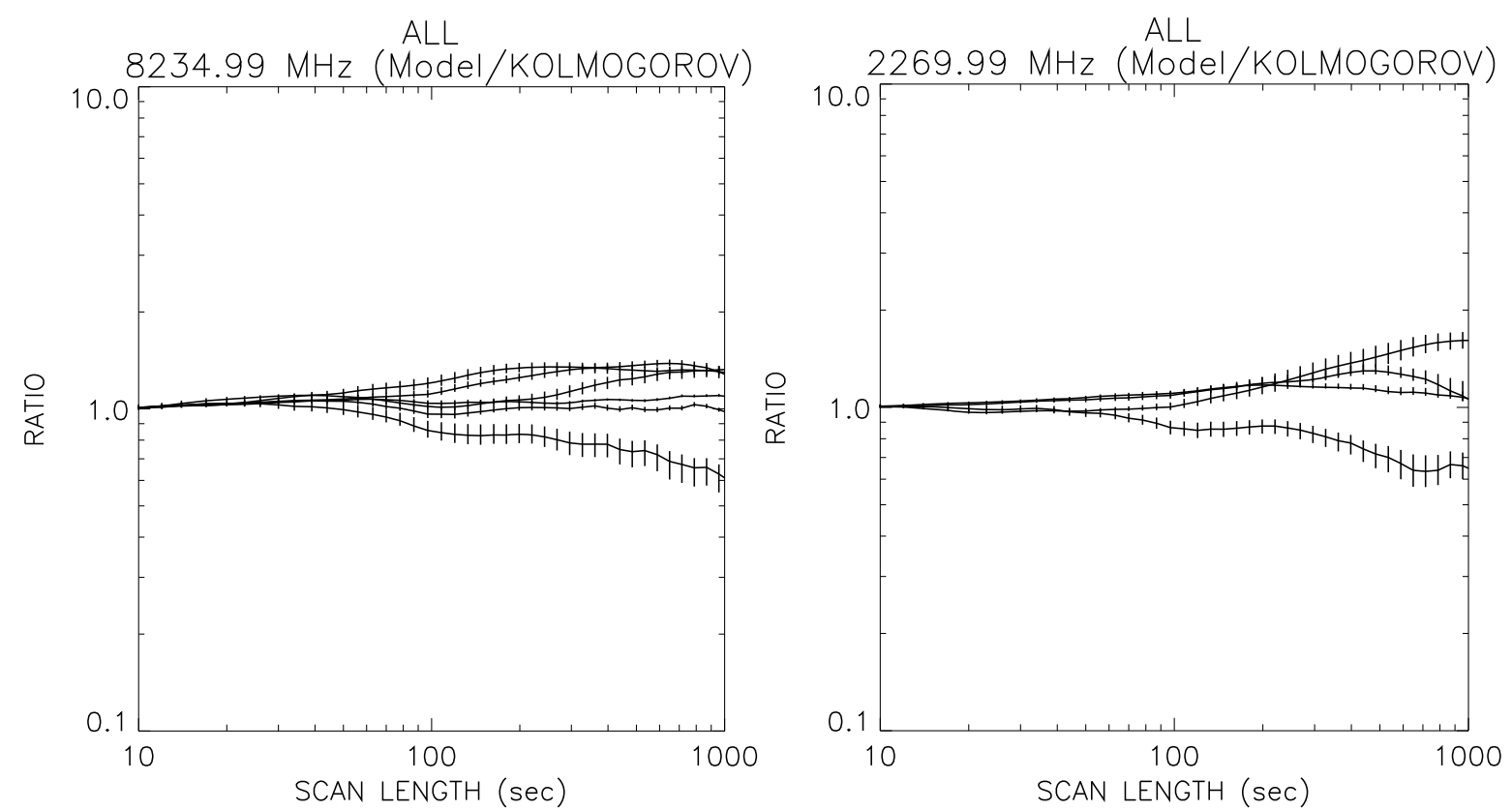

Fig. 12. Same as Fig. 7 but for ratios of standard deviation of phase errors obtained from actual phase structure functions and those obtained by Kolmogorov model fitting for $8234.99 \mathrm{MHz}$ (left panel) and for 2269.99 MHz (right panel).

A4) Assuming a normal distribution variable (thermal noise) only to express an SNR integrated over the whole scan length in Eq. (15);

A5) Obtaining $\sigma_{\phi}$ from a phase structure function at the shortest time scale in Eq. (17).

Regarding A1, we have computed the magnitude of cross-terms and the ratio to the total term to check the validity of this assumption. The last term of the right hand side of Eq. (7) sometimes shows a ratio exceeding 0.5; however, the root of absolute value is less than $30^{\circ}$ for most of the combinations of scan and gap lengths. Therefore, this as- sumption can affect the results by about a few tens of degrees. A2 usually underestimates a fitting error at the end of the scan period for actual phase fluctuations at the time scale concerned in this study.

A3 gives the overestimation in the case of a normal distribution variable. A4 overestimates an SNR for actual phase fluctuations. A5 overestimates $\sigma_{\phi}$ because a phase structure function at the shortest time scale (now it is $1 \mathrm{~s}$ ) may include the fluctuations due to the propagation media even though it is thought to be small enough.

The validity of the model derived from these assumptions has been proven by comparisons with the actual linear fit- 

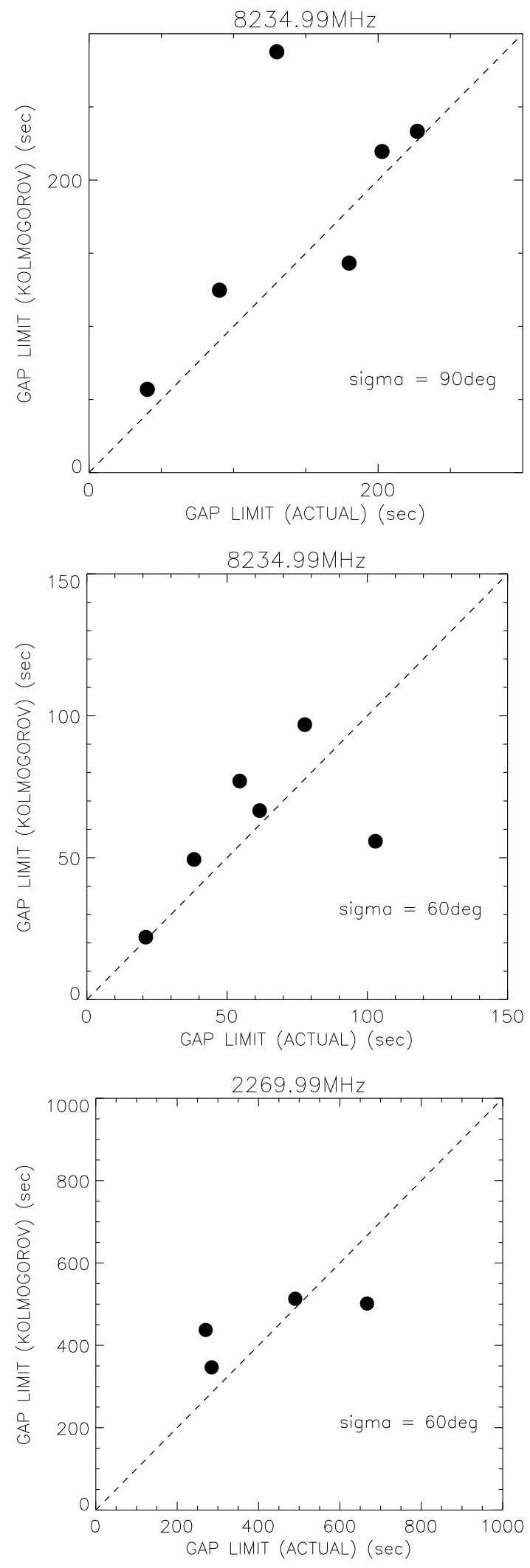

Fig. 13. Scatter plots of gap limits obtained from Kolmogorov model and actual linear fitting for all sessions. Top and middle panels are for $8234.99 \mathrm{MHz}$ and bottom for 2269.99 MHz. Standard deviations of $90^{\circ}$ (top panel) and $60^{\circ}$ (middle and bottom panels) are adopted as thresholds to calculate a gap limit under the condition of gap length $\left(t_{\mathrm{g}}\right)=$ scan length $\left(t_{\mathrm{s}}\right)$. Plots of the case of standard deviations of $90^{\circ}$ for $2269.99 \mathrm{MHz}$ are omitted because all available gap lengths exceed $1000 \mathrm{~s}$. ting results, as shown in Figs. 5 and 6. Their coincidence is demonstrated in Fig. 7, which plots their ratio averaged over gap lengths of between 10 to $1000 \mathrm{~s}$ as a function of scan length, with vertical bars indicating the range of the ratio. We can see a good correspondence, particularly for X-band $(8234.99 \mathrm{MHz})$ at periods shorter than $600 \mathrm{~s}$. However, for most cases, the ratio is larger than 1.0 and sometimes exceeds 2.0 for the S-band (2269.99 MHz), i.e., the standard deviation of phase error estimated from the simple model is larger than that obtained from the actual linear fitting. Therefore, the available scan gap is estimated to be shorter than the actual one. In other words, the available gap length is underestimated in comparison with the real one, so that it will ensure the correct connection of phase delay.

Although the model has some defects, it is concluded that we can use Eq. (18) for evaluating the relation between scan length and available gap length from a phase structure function.

The next issue to discuss is how accurately we can estimate phase structure functions at longer time periods from those at a shorter time. As shown in Fig. 8, actual phase structure functions at a time period of less than $100 \mathrm{~s}$, especially $10-100 \mathrm{~s}$, are well-characterized by a fixed slope of about 1.0 on the log-log plot. The same quantitative characteristics can be seen in Fig. 8 of Liu et al. (2005). Thus, it is expected that the estimation of a whole structure function from that at $10 \mathrm{~s}$ will give a reasonable phase structure function. Actually, as shown in Fig. 9, the whole structure function shows a good correspondence with an observed one. Relative errors are within \pm 0.5 at $10-1000$ s except for four lines which correspond to two S-band and two X-band data observed on March 16, 2006 under rain conditions at both stations. Structure functions observed on March 16 show slightly different features than the others, i.e., the slope on the log-log plot at a large time scale is more moderate than the others, and transition to this moderate slope occurs at a time scale shorter than that of the others. This results in the large discrepancy at the large time scale. The results of standard deviations of $\Delta \phi$ calculated by an estimated phase structure function is shown in Fig. 10. They coincide well with those obtained by actual linear fittings (Fig. 6). Their ratio is plotted in Fig. 11 with the same format as Fig. 7.

We can see similar characteristics with Fig. 7. From this result, it can be expected that the standard deviations calculated from an actual phase structure function and those based on Kolmogorov model fitting at a short time period coincide well with each other. Actually, their difference is less than a factor of 2 at time periods $10-1000 \mathrm{~s}$, as shown in Fig. 12. We therefore conclude that we can infer a fairly accurate phase structure function at longer time periods by using $\sigma^{2}(\tau)$ at $\tau=10 \mathrm{~s}$ and we can use it to estimate standard deviations of $\Delta \phi$.

Figure 13 shows scatter plots of gap limits obtained from the Kolmogorov model and actual linear fitting for 8234.99 MHz and 2269.99 MHz for all sessions. Standard deviations of $90^{\circ}$ (corresponding to connection of phases without ambiguities with a probability of $95 \%$ ) and $60^{\circ}$ (99.7\% probability) are adopted as thresholds to calculate a gap limit under the condition of gap length $\left(t_{\mathrm{g}}\right)=$ scan length $\left(t_{\mathrm{s}}\right)$, but plots of the case of standard deviations of 

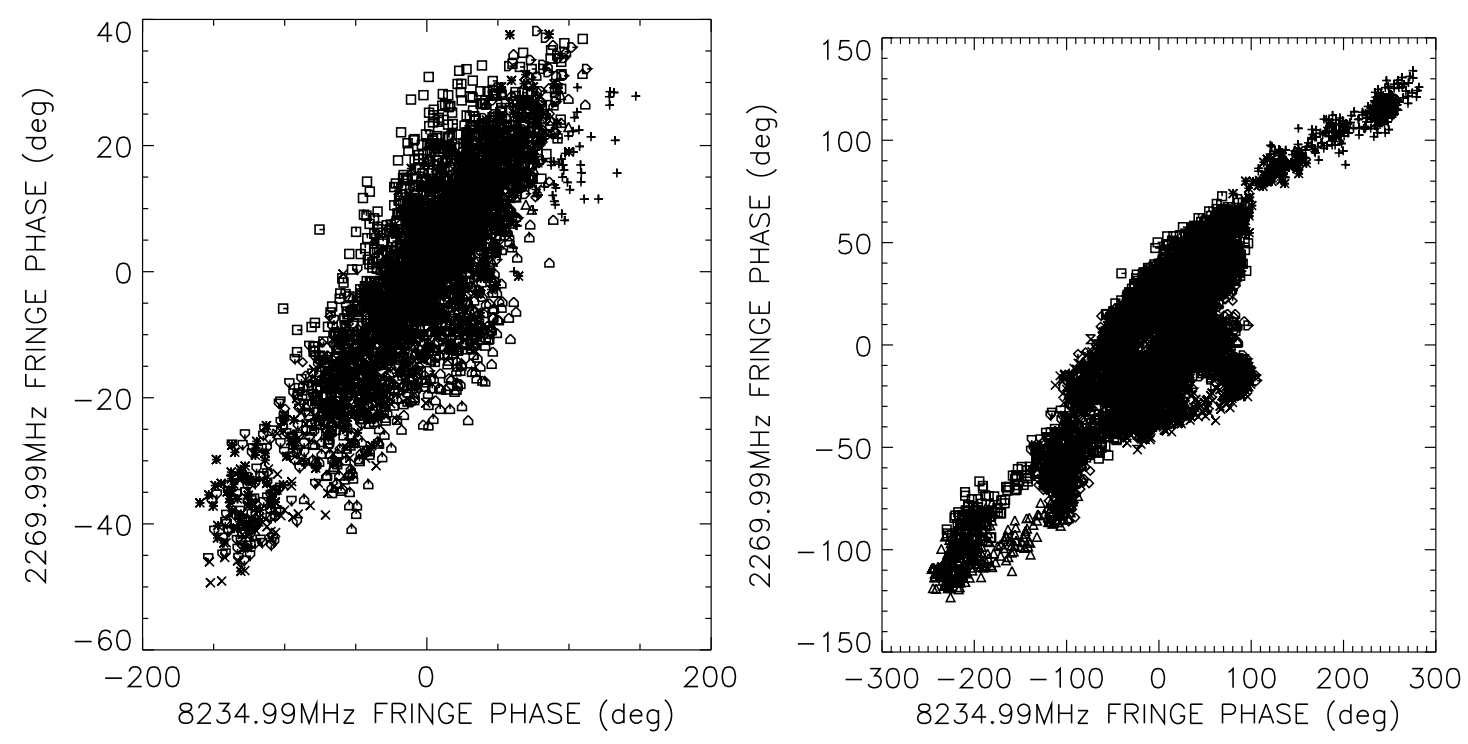

Fig. 14. Scatter plots of fringe phases of $8234.99 \mathrm{MHz}$ and 2269.99 MHz observed on March 16 (left panel) and on May 11 (right panel), 2006.

$90^{\circ}$ for $2269.99 \mathrm{MHz}$ are omitted because all available gap lengths exceed $1000 \mathrm{~s}$. As shown in this figure, both limits coincide fairly well with each other. Actual gap limits vary significantly session by session from about 50-230 s for a standard deviation of $90^{\circ}$ and $20-110 \mathrm{~s}$ for $60^{\circ}$ at 8234.99 MHz. As for $2269.99 \mathrm{MHz}$, they exceed $1000 \mathrm{~s}$ for $90^{\circ}$ and $250-700 \mathrm{~s}$ for $60^{\circ}$. The range of gap limits in the case of a standard deviation of $90^{\circ}$ is consistent with Beasley and Conway's (1995) prediction of 160-440 s for switching time (that is about double the gap length) at a frequency of $8.4 \mathrm{GHz}$, which is based on the $90^{\circ}$ criterion. They are also consistent with the VLBA's recommendation on a phase referencing observation at frequencies 1.4$8.4 \mathrm{GHz}$, which is a switching time of $300 \mathrm{~s}(180 \mathrm{~s}$ on a target and $120 \mathrm{~s}$ on a calibrator).

As shown in Fig. 13, available gap lengths for the S-band are usually longer than those for the X-band. The delay fluctuations caused by the troposphere are independent of frequency, while those caused by the ionosphere are written inversely as the square of the frequencies. We usually see a good positive correlation between S-band phase fluctuations and X-band ones, as shown in the left panel of Fig. 14. The magnitude of the fluctuations of the S-band is about one-fourth of that of the X-band, so that it can be concluded that such fluctuations are caused by the non-dispersive delay change due to the troposphere. We therefore consider that the idea of connecting X-band data using phase delay data observed for S-band will work well for this case. However, there is a case which indicates hysteresis-like fluctuations and suggests the existence of uncorrelated components, as shown in the right panel of Fig. 14. Instrumentation, ionosphere, and solar wind plasma can cause such uncorrelated components. In particular, scintillations caused by the solar wind affects phase fluctuations at an S-band larger than those at the X-band. An area affected by large scintillations (scintillation index $>0.1$ ) at the S-band exceeds an angle distance of $10^{\circ}$ from the sun at solar maximum (Tokumaru et al., 1995). Therefore, the connection of fringe phases at the $\mathrm{X}$-band using S-band fringe phase data is thought not to work adequately in some cases, such as in the case of solar maximum at an area close to the sun. This issue, although very interesting, is far beyond the scope of this paper and will not be discussed here.

In the case of D-VLBI, which observes two celestial radio sources alternately, a scan period for a source corresponds to a gap period for the other source. In practice, the time of antenna motion necessary for source change should be considered as a part of the gap period. Let $t_{\mathrm{c}}$ be a time required to change the source, a necessary condition to be satisfied by a gap length $t_{\mathrm{g}}$ is given by

$$
t_{\mathrm{g}} \geq t_{\mathrm{s}}+2 t_{\mathrm{c}} \text {. }
$$

A solution can be obtained, for example, graphically by plotting Eq. (26) on Fig. 10. However, we will not discuss these results here because the purpose of this study is to evaluate the possibility of scan-gap limit from a structure function obtained at a short time period.

We have discussed the possibility of estimating a phase structure function at longer time periods by using observed one at $\tau=10 \mathrm{~s}$. There are many factors that influence a phase structure function, such as weather condition, ionosphere condition, source elevation angle, SNR noise, etc., but they are thought to be reflected in a phase structure function at a short time period. Therefore, although the number of sessions analyzed in our study is only five, which may be insufficient to discuss statistically the characteristics of the magnitude of phase fluctuations under various weather conditions, we think that the data of one session, that spanning 3600-7200 s, are sufficient to discuss the statistical characteristics of a phase structure function at the various time periods emphasized in this study. In order to confirm the validity of the method proposed in this study, a statistical study using a number of session data under the various weather conditions and seasons is necessary. Such a study will clarify the characteristics of the phase structure functions under the various conditions and will help construct a better model. We would like to leave this challenges to future researchers. 
Lastly, we discuss about an accuracy requirement.

According to Thornton and Border (2003), a requirement for the angular position measurement of a spacecraft is $50 \mathrm{nrad}$ during interplanetary cruise phases and 5-10 nrad to deliver landers on the surface of Mars. For example, $5 \mathrm{nrad}$ as a requirement for the measurement accuracy corresponds to a one-sigma delay error of 5 ps for a projected baseline length of $300 \mathrm{~km}$ (close to Kashima-Gifu baseline length). This is not a difficult requirement even for narrow bandwidth signals from a spacecraft if we can measure a phase delay without ambiguities, because 5 ps corresponds to $14.4^{\circ}$ at $8 \mathrm{GHz}$ and it is easily achieved by observations of $S N R>4$ (see Eq. (14)). However, this is for an ideal case in which common error sources, such as those introduced by receiving systems, clocks, propagating media, and station locations, can be completely cancelled by D-VLBI, and phase delays can be connected without ambiguities among consecutive scans over a gap.

In other words, we suggest that it is possible to measure an angular position of spacecraft by D-VLBI on a short baseline, such as $300 \mathrm{~km}$, with an accuracy meeting with a requirement for a Mars lander if the use of phase delay is realized through the phase delay connection among consecutive scans.

Discussions on the accuracy requirement described above are for the case that the phase-delay connection is achieved perfectly without any ambiguities. Failure in the phase-delay connection between consecutive scans introduces a bias error equal to an ambiguity in observed delay (we suppose minimum ambiguity here for simplicity). An effective bias error will be given by a simple weighted mean regarding the number of scans accompanied by an ambiguity. However, we do not know where the phase-delay connection fails in the case of (i.e., one-baseline) observations made at two stations (a failure in phase-delay connection may be found through the "closure phase" method in the case observations made at three or more stations). If the phase-delay connection fails at the last scan, an effective bias error will be small. If it fails at the second scan, an effective bias error keeps a large value close to an ambiguity. Although an effective bias varies in this way, we can consider the half of ambiguity as an effective bias error when the phase-delay connection fails only once in a series of scans. An ambiguity is $125 \mathrm{ps}$ at $8 \mathrm{GHz}$, so that an effective bias can be considered as $62.5 \mathrm{ps}$, and it is larger than the required accuracy on the short baseline described above. Let the probability of failure in the phase-delay connection be $\mathrm{P}$, and the number of scans where phase-delay connection fails is thought to be given by $\mathrm{PN}$ where $\mathrm{N}$ is the total number of scans. $\mathrm{P}$ is $5 \%$ for the $90^{\circ}$ criterion and $0.3 \%$ for the $60^{\circ}$ criterion. Therefore, 20 scans include at least one scan where the phase-delay connection fails in the case of the $90^{\circ}$ criterion and 333 scans for the $60^{\circ}$ criterion. Based on actual available gap limits (50-230 s for the $90^{\circ}$ criterion and $20-110 \mathrm{~s}$ for the $60^{\circ}$ criterion at $8234.99 \mathrm{MHz}$ ), the total scan number can exceed 20 in the case of the $90^{\circ}$ criterion. Thus, the $60^{\circ}$ criterion is thought to be more preferable for an actual application.

\section{Conclusions}

We have carried out a series of VLBI experiments in 2006 using three stations, Kashima, Gifu, and Koganei to investigate the relation between scan length and scan gap limit.

Our first step has been to develop a simple method to evaluate a scan gap limit based on the error analysis of linear fitted data using a phase structure function. This has been derived from a simple assumption regarding the error of phase changing rate, i.e., the error is described only by the phase difference at the both ends of scan period. Standard deviations of phase errors over a gap estimated by this method have been compared with those estimated from an actual linear fitting of fringe phases, and the validity of the simple method developed in this study has been confirmed. However, in most cases, the available scan gap estimated from the simple model is a bit shorter than the actual one. This means that the improvement of the model will give a more correct scan-gap length, but we leave this for a future study.

As for the possibility of the estimation of a whole structure function from that at a short time period, which is necessary for dynamic scheduling at D-VLBI, a model based on the Kolmogorov turbulence theory has been proposed. We model a phase structure function depending on the wet atmosphere by using a piecewise linear function on the log$\log$ plot; a phase structure function follows a power law having three different slopes $1.67(=5 / 3), 1.00$, and 0.67 $(=2 / 3)$ for three time ranges, 1-10, 10-100, and 100$1000 \mathrm{~s}$, respectively. Since the function is continuous at boundaries, the model can estimate a structure function at $\tau>100 \mathrm{~s}$ from that at $\tau=10 \mathrm{~s}$. The validity of the model has been confirmed by a comparison of standard deviations of phase errors over a gap estimated from a structure function obtained by this model and those estimated from an actual linear fitting of fringe phases. Hence, it can be concluded that we can estimate a fairly accurate phase structure function at longer time periods by using that at $\tau=10 \mathrm{~s}$, and we can use it to estimate standard deviations of phase error over a scan gap. This suggests that it is possible to control scan length and gap length dynamically in order to achieve the best performance for D-VLBI observations.

Acknowledgments. A part of this work was carried out during a temporary stay by one of authors (TK) at the Vienna University of Technology (TU). He thanks Harald Schuh, head of the Institute of Geodesy and Geophysics, TU, for supporting his stay. This research was supported in part by the research project P16136N06 "Investigation of the ionosphere by geodetic VLBI" founded by the Austrian Science Fund (FWF).

\section{References}

Beasley, A. J. and J. E. Conway, VLBI phase-referencing, in Very Long Baseline Interferometry and the VLBA, ASP Conf. Ser. 82, edited by $\mathrm{J}$. A. Zensus, P. J. Diamond, and P. J. Napier, 327-343, 1995.

Border, J. S. and J. A. Koukos, Technical characteristics and accuracy capabilities of delta differential one-way ranging $(\triangle D O R)$ as a spacecraft navigation tool, CCSDS B20.0-Y-1, Report of the Proceedings of the $R F$ and Modulation Subpanel $1 E$ Meeting at the German Space Operations Centre: September 20-24, 1993, edited by T. M. Nguyen, Yellow Book, Oberpfaffenhofen, Germany: Consultative Committee for Space Data Systems, February, 1994.

Brunn, D. L., R. A. Preston, S. C. Wu, H. L. Siegel, D. S. Brown, C. 
S. Christensen, and D. E. Hilt, Delta VLBI spacecraft tracking system demonstration: Part I. design and planning, DSN Progress Report 42-45, 111-132, 1978.

Christensen, C. S., B. Moultrie, P. S. Callahan, F. F. Donivan, and S. C. Wu, Delta VLBI spacecraft tracking system demonstration: Part II: data acquisition and processing, TDA Progress Report 42-60, 60-67, 1980.

Fujiwara, A., T. Mukai, J. Kawaguchi, and K. T. Uesugi, Sample return mission to NEA: MUSES-C, Advances in Space Research, 25, 231-238, 1999.

Ichikawa, R., M. Sekido, H. Osaki, Y. Koyama, T. Kondo, T. Ohnishi, M. Yoshikawa, W. Cannon, A. Novikov, M. Berube, and NOZOMI DVLBI GROUP, An evaluation of VLBI observations for the deep space tracking on the interplanetary spacecrafts, in International VLBI Service for Geodesy and Astrometry 2004 General Meeting Proceedings, edited by Nancy R. Vandenberg and Karen D. Baver, NASA/CP-2004-212255, 253-257, 2004.

Ichikawa, R., M. Sekido, Y. Koyama, and T. Kondo, An evaluation of atmospheric path delay correction in differential VLBI experiments for spacecraft tracking, in International VLBI Service for Geodesy and Astrometry 2006 General Meeting Proceedings, edited by D. Behrend and K. D. Baver, NASA/CP-2006-214140, 226-230, 2006.

Kasuga, T., M. Ishiguro, and R. Kawabe, Interferometric measurement of tropospheric phase fluctuations at $22 \mathrm{GHz}$ on antenna spacings of 27 to 540 m, IEEE Trans. Antennas Propag., AP-34, 797-803, 1986.

Kikuchi, F., Y. Kono, M. Yoshikawa, M. Sekido, M. Ohnishi, Y. Murata, J. Ping, Q. Liu, K. Matsumoto, K. Asari, S. Tsuruta, H. Hanada, and N. Kawano, VLBI observation of narrow bandwidth signals from the spacecraft, Earth Planets Space, 56, 1041-1047, 2004.

Kondo, T., Y. Koyama, J. Nakajima, M. Sekido, and H. Osaki, Internet VLBI system based on the PC-VSSP (IP-VLBI) board, in New Technologies in VLBI, ASP Conf. Ser. 306, edited by Minh, Y. C., 205-216, 2003.

Kondo, T., M. Kimura, Y. Koyama, and H. Osaki, Current status of software correlators developed at Kashima Space Research Center, in International VLBI Service for Geodesy and Astrometry 2004 General Meeting Proceedings, edited by Nancy R. Vandenberg and Karen D. Baver, NASA/CP-2004-212255, 186-190, 2004.

Kono, Y., H. Hanada, J. Ping, Y. Koyama, Y. Fukuzaki, and N. Kawano, Precise positioning of spacecrafts by multi-frequency VLBI, Earth Planets Space, 55, 581-589, 2003.

Koyama, Y., T. Kondo, M. Kimura, H. Takeuchi, and M. Hirabaru, e-VLBI developments with the K-5 system, in International VLBI Service for Geodesy and Astrometry 2006 General Meeting Proceedings, edited by D. Behrend and K. D. Baver, NASA/CP-2006-214140, 216-220, 2006. Liu, Q., M. Nishio, K. Yamamura, T. Miyazaki, M. Hirata, T. Suzuyama, S. Kuji, K. Iwadate, O. Kameya, and N. Kawano, Statistical characteristics of atmospheric phase fluctuations observed by a VLBI system using a beacon wave from a geostationary satellite, IEEE Trans. Antennas Propag., AP-53, 1519-1527, 2005.

Rogers, A. E. E., Very-long-baseline interferometry with large effective bandwidth for phase-delay measurements, Radio Sci., 5, 1239-1247, 1970 .

Rogers, A. E. E. and J. M. Moran, Coherence limit for very long baseline interferometry, IEEE Trans. Instrum. Meas., IM-30, 283-286, 1981.

Rogers, A. E. E., A. T. Moffet, D. C. Backer, and J. M. Moran, Coherence limits in VLBI observation at 3-millimeter wave length, Radio Sci., 19, 1552-1560, 1984.

Sekido, M., R. Ichikawa, H. Osaki, T. Kondo, Y. Koyama, M. Yoshikawa, T. Ohnishi, W. Cannon, A. Novikov, M. Berube, and NOZOMI DVLBI GROUP, VLBI observation spacecraft navigation (NOZOMI)_data processing and analysis status report, in International VLBI Service for Geodesy and Astrometry 2004 General Meeting Proceedings, edited by Nancy R. Vandenberg and Karen D. Baver, NASA/CP-2004-212255, 258-262, 2004.

Takahashi, F., T. Kondo, Y. Takahashi, and Y. Koyama, Very long baseline interferometer, Ohmsha, Tokyo, 2000.

Tatarskii, V. I., Wave Propagation in a Turbulent Medium, Dover, New York, 1961.

Thornton, C. L. and J. S. Border, Radiometric tracking techniques for deep-space navigation, John Wiley \& Sons. Inc., Hoboken, New Jersey, 2003.

Tokumaru, M., H. Mori, T. Tanaka, and T. Kondo, Evolution of the Solar Wind Structure in the Acceleration Region during (STEP Interval) 1990-1993, J. Geomag. Geoelectr., 47, 1113-1120, 1995.

Treuhaft, R. N. and G. E. Lanyi, The effect of the dynamic wet troposphere on radio interferometric measurements, Radio Sci., 22, 251-265, 1987.

Whitney, A. R. and C. A. Ruszczyk, e-VLBI development at Haystack Observatory, in International VLBI Service for Geodesy and Astrometry 2006 General Meeting Proceedings, edited by D. Behrend and K. D. Baver, NASA/CP-2006-214140, 211-215, 2006.

Wrobel, J. M., R. C. Walker, J. M. Benson, and A. J. Beasley, VLBA scientific memorandum 24: Strategies for phase referencing with the VLBA, http://www.vlba.nrao.edu/memos/sci/sci24memo.ps, 2000.

$\mathrm{Wu}, \mathrm{S}$. C., Connection and validation of narrow-band delta VLBI phase observations, DSN Progress report 42-52, 13-20, 1979.

Yamamoto, T. and K. Tsuruda, The PLANET-B mission, Earth Planets Space, 50, 175-181, 1998.

T. Kondo (e-mail: kondo@nict.go.jp), T. Hobiger, M. Sekido, R. Ichikawa, Y. Koyama, and H. Takaba 